\title{
A Spatial Cliff-Ord-type Model with Heteroskedastic Innovations: Small and Large Sample Results
}

\author{
IRANI ARRAIZ \\ DAVID M. DRUKKER \\ HARRY H. KELEJIAN \\ INGMAR R. PRUCHA
}

CESIFO WORKING PAPER NO. 2485

CATEGORY 10: EMPIRICAL AND THEORETICAL METHODS

DECEMBER 2008

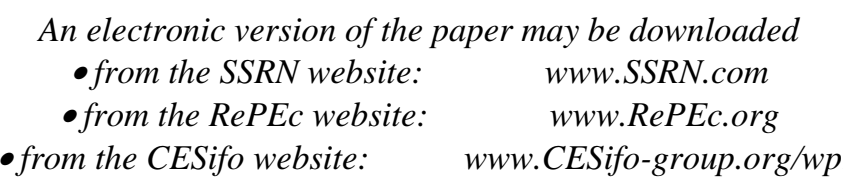




\title{
A Spatial Cliff-Ord-type Model with Heteroskedastic Innovations: Small and Large Sample Results
}

\begin{abstract}
In this paper we specify a linear Cliff and Ord-type spatial model. The model allows for spatial lags in the dependent variable, the exogenous variables, and disturbances. The innovations in the disturbance process are assumed to be heteroskedastic with an unknown form. We formulate a multi-step GMM/IV type estimation procedure for the parameters of the model. We then establish the limiting distribution of our suggested estimators, and give consistent estimators for their asymptotic variance covariance matrices, utilizing results given in Kelejian and Prucha (2007b). Monte Carlo results are given which suggest that the derived large sample distribution provides a good approximation to the actual small sample distribution of our estimators.
\end{abstract}

JEL Code: C21, C31.

\author{
Irani Arraiz \\ Inter-American Development Bank \\ 1300 New York Ave. \\ USA - N.W., Washington, DC 20577 \\ arraiz@econ.bsos.umd.edu \\ Harry H. Kelejian \\ Department of Economics \\ University of Maryland \\ USA - College Park, MD 20742 \\ kelejian@econ.umd.edu
}

\author{
David M. Drukker \\ StataCorp \\ 4905 Lakeway Drive \\ USA - College Station, TX 77845 \\ ddrukker@stata.com \\ Ingmar R. Prucha \\ Department of Economics \\ University of Maryland \\ USA - College Park, MD 20742 \\ prucha@econ.umd.edu
}

September 20, 2007

Our thanks for very helpful comments are owed to Peter Egger, Michael Pfaffermayr, and Gianfranco Piras. Also, we gratefully acknowledge financial support from the National Institute of Health through the SBIR grant 1 R43 AG027622. Ingmar Prucha also thanks the CESifo in Munich for their hospitality and appreciates their support in writing this paper. 


\section{Introduction}

Spatial econometric models, which are variants of the ones suggested by Cliff and Ord $(1973,1981)$, have been widely used in regional science, geography, and economics. ${ }^{1}$ Maximum likelihood is one method of estimating the parameters of these models. However, until Lee (2004), there was no formal theory establishing large-sample results for maximum-likelihood estimators of the parameters of these models. In addition, as Kelejian and Prucha (1999) indicate, under certain conditions the implementation of maximum-likelihood procedures involves computational difficulties. Kelejian and Prucha (1999) suggested an alternative method of estimating these models to overcome these hurdles. Their procedure was based on a generalized method-of-moments (GMM) estimator of the autoregressive parameter in the disturbance process. Although they demonstrated the consistency of their GMM estimator, they did not determine its large-sample distribution and so tests relating to that autoregressive parameter could not be carried out in their framework. Kelejian and Prucha (1999) gave their results under the usual assumption that the innovations of the disturbance process were homoskedastic. This homoskedasticity assumption restricts the scope of applications of their procedure because cross-sectional spatial units often differ in size and other characteristics which causes one to suspect that the innovations to the disturbance process are heteroskedastic.

In a later paper, Kelejian and Prucha (2007b) extended their earlier results in a variety of directions. In particular, they considered a Cliff-Ord-type spatial-autoregressive process with heteroskedastic innovations and suggested a GMM estimator, say $\hat{\rho}$, for the autoregressive parameter of that process. That GMM estimator was assumed to be based on estimated residuals that were formulated in terms of a regression parameter estimator, say $\hat{\delta}$. Under reasonably general conditions, they gave the large-sample distribution of their GMM estimator, $\hat{\rho}$. They also gave the joint large-sample distribution of their GMM estimator $\hat{\rho}$ and the regression parameter estimator $\hat{\delta}$.

Given their generality the results in Kelejian and Prucha (2007b) cover a wide array of settings. For example, their assumptions underlying their GMM estimator $\hat{\rho}$ were general enough to include cases in which the residuals that were used in their GMM estimation could have come from a linear, or a non-linear spatial regression model. Similarly, their results concerning the large-sample joint distribution of their GMM estimator $\hat{\rho}$ and the regression parameter estimator $\hat{\delta}$ were general enough to include a variety of cases. For example, their regression parameter estimator could be a 2SLS estimator, a feasible generalized 2SLS estimator, etc. Similarly, their GMM estimator $\hat{\rho}$ could be based on estimated residuals obtained from a 2SLS estimator, a feasible generalized-spatial 2SLS estimator (GS2SLS), etc. Their results

\footnotetext{
${ }^{1}$ Classic references to spatial models are Cliff and Ord (1973, 1981), Anselin (1988), and Cressie (1993). Some recent applications are Baltagi, Egger, and Pfaffermayr (2007), Cohn and Morrison Paul (2003, 2004, 2007), Keller and Shiue (2007), Anselin and Le Gallo (2006), Le Gallo and Dall'erba (2006), Hanushek et al. (2003), Topa (2001), Sacerdote (2001), Betrand, Luttmer and Mullainathan (2000), Bell and Bockstael (2000), Audretsch and Feldmann (1996), Besley and Case (1995), Shroder (1995), Holtz-Eakin (1994), and Case, Hines, and Rosen (1993).
} 
concerning the joint large-sample distribution of $\hat{\rho}$ and $\hat{\delta}$ were general enough to cover a variety of combinations of $\hat{\rho}$ and $\hat{\delta}$. For instance, among other things, $\hat{\rho}$ could be based on 2SLS residuals, and $\hat{\delta}$ could be the GS2SLS estimator.

Given their aim of providing a general estimation theory, Kelejian and Prucha (2007b) do not provide specific expressions for the large-sample distribution for specific estimators. Because of this practitioners may find it challenging and/or tedious to specialize the general distributional results for a particular estimator-model combination.

The purpose of this paper is three fold. First, we specify a typical linear spatial model that might be considered in practice and demonstrate that our suggested estimators of its parameters satisfy the general assumptions in Kelejian and Prucha (2007b). This model allows for spatial lags in the dependent variable, the exogenous variables, and disturbances, and allows for heteroskedasticity of unknown form in the innovations. Second, we specialize the general distributional results in Kelejian and Prucha (2007b) for our estimators of the parameters in this model. These results make estimation of and inference about the parameters of this spatial model, and special cases of it, straight forward. Third, we give Monte Carlo results which describe the small-sample properties of our estimators, the estimators of their variances, as well as corresponding Wald-type tests.

Our Monte Carlo results suggest that our estimators behave quite nicely in small samples. They also suggest that the maximum-likelihood estimator of the autoregressive parameter in the disturbance process can be substantially biased in certain circumstances.

\section{A Spatial Cliff-Ord-Type Model}

\section{$2.1 \quad$ Specifications}

In this section we specify a linear spatial model that allows for spatial lags in the dependent variable, the exogenous variables, and disturbances. Consistent with the terminology introduced by Anselin (1988), and used elsewhere in the literature, e.g. in Kelejian and Prucha (2007a), we refer to this model as a spatial $\operatorname{ARAR}(1,1)$ model, i.e., $\operatorname{SARAR}(1,1)$. The specification does not assume homoskedastic innovations, but instead allows for heteroskedasticity of unknown form. Apart from allowing for heteroskedasticity the assumptions are similar to those made in the existing literature. Since those assumptions have been discussed in detail before, our discussion of them will be brief. ${ }^{2}$

Consider the following spatial model relating to $n$ cross sectional units:

$$
\begin{aligned}
y_{n} & =X_{n} \beta+\lambda W_{n} y_{n}+u_{n} \\
& =Z_{n} \delta+u_{n},
\end{aligned}
$$

and

$$
u_{n}=\rho M_{n} u_{n}+\varepsilon_{n},
$$

\footnotetext{
${ }^{2}$ Among other studies, see Kelejian and Prucha (1998, 2004, 2007a,b) for a more extensive discussion of these assumptions.
} 
where $Z_{n}=\left[X_{n}, W_{n} y_{n}\right], \delta=\left[\beta^{\prime}, \lambda\right]^{\prime}, y_{n}$ is the $n \times 1$ vector of observations of the dependent variable, $X_{n}$ is the $n \times k$ matrix of observations on non-stochastic (exogenous) regressors, $W_{n}$ and $M_{n}$ are $n \times n$ non-stochastic weights matrices, $u_{n}$ is the $n \times 1$ vector of regression disturbances, $\varepsilon_{n}$ is an $n \times 1$ vector of innovations, $\lambda$ and $\rho$ are scalar parameters, and $\beta$ is a $k \times 1$ vector of parameters. The subscript $n$ denotes dependence on the sample size and so (1) and (2) allow for triangular arrays. Consequently, this specification allows some or all of the exogenous variables to be spatial lags of exogenous variables. Thus the model is fairly general in that it allows for spatial spill-overs in the endogenous variables, exogenous variables, and disturbances.

Our discussions will also utilize the following spatial Cochrane-Orcutt transformation of (1) and (2):

$$
y_{n *}(\rho)=Z_{n *}(\rho) \delta+\varepsilon_{n},
$$

where $y_{n *}(\rho)=y_{n}-\rho M_{n} y_{n}$ and $Z_{n *}(\rho)=Z_{n}-\rho M_{n} Z_{n}$. The transformed model is readily obtained by pre-multiplying (1) by $I_{n}-\rho M_{n}$.

The spatial weights matrices and the autoregressive parameters are assumed to satisfy the following assumption.

Assumption 1 (a) All diagonal elements of $W_{n}$ and $M_{n}$ are zero. (b) $\lambda \in$ $(-1,1), \rho \in(-1,1)$. (c) The matrices $I_{n}-\bar{\lambda} W_{n}$ and $I_{n}-\bar{\rho} M_{n}$ are nonsingular for all $\bar{\lambda} \in(-1,1)$, and $\bar{\rho} \in(-1,1)$.

Assumption 2 The innovations $\left\{\varepsilon_{i, n}: 1 \leq i \leq n, n \geq 1\right\}$ satisfy $E \varepsilon_{i, n}=0$, $E\left(\varepsilon_{i, n}^{2}\right)=\sigma_{i, n}^{2}$ with $0<\underline{a}^{\sigma} \leq \sigma_{i, n}^{2} \leq \bar{a}^{\sigma}<\infty$, and $\sup _{1 \leq i \leq n, n \geq 1} E\left|\varepsilon_{i, n}\right|^{4+\eta}<$ $\infty$ for some $\eta>0$. Furthermore, for each $n \geq 1$ the random variables $\varepsilon_{1, n}, \ldots, \varepsilon_{n, n}$ are totally independent.

Assumption 3 The row and column sums of the matrices $W_{n}$ and $M_{n}$ are bounded uniformly in absolute value by, respectively, one and some finite constant, and the row and column sums of the matrices $\left(I_{n}-\lambda W_{n}\right)^{-1}$ and $\left(I_{n}-\rho M_{n}\right)^{-1}$ are bounded uniformly in absolute value by some finite constant.

It is evident from (1) and (2) that, under typical specifications, $W_{n} y_{n}$ will be correlated with the disturbances $u_{n}$, which motivates the use of the instrumental variable procedure. The selection of instruments as an approximation to ideal instruments is discussed in Kelejian and Prucha (1998, 2007a,b), and a review of that discussion is given below. At this point let $H_{n}$ be an $n \times p$ matrix of non-stochastic instruments where $p \geq k+1$, and note that in practice $H_{n}$ would depend upon $X_{n}$. Our assumptions concerning $X_{n}$ and $H_{n}$ are given below. 
Assumption 4 : The regressor matrices $X_{n}$ have full column rank (for $n$ large enough). Furthermore, the elements of the matrices $X_{n}$ are uniformly bounded in absolute value.

Assumption 5 : The instrument matrices $H_{n}$ have full column rank $p \geq$ $k+1$ (for all $n$ large enough). Furthermore, the elements of the matrices $H_{n}$ are uniformly bounded in absolute value. Additionally $H_{n}$ is assumed to, at least, contain the linearly independent columns of $\left(X_{n}, M_{n} X_{n}\right)$.

Assumption 6 : The instruments $H_{n}$ satisfy furthermore:

(a) $Q_{H H}=\lim _{n \rightarrow \infty} n^{-1} H_{n}^{\prime} H_{n}$ is finite, and nonsingular.

(b) $Q_{H Z}=\operatorname{plim}_{n \rightarrow \infty} n^{-1} H_{n}^{\prime} Z_{n}$ and $Q_{H M Z}=\operatorname{plim}_{n \rightarrow \infty} n^{-1} H_{n}^{\prime} M_{n} Z_{n}$ are finite and have full column rank. Furthermore, $Q_{H Z *}(\rho)=Q_{H Z}-\rho Q_{H M Z}$ has full column rank.

(c) $Q_{H \boldsymbol{\Sigma} H}=\lim _{n \rightarrow \infty} n^{-1} H_{n}^{\prime} \boldsymbol{\Sigma}_{n} H_{n}$ is finite and nonsingular, where $\boldsymbol{\Sigma}_{n}=$ $\operatorname{diag}_{i=1}^{n}\left(\sigma_{i, n}^{2}\right)$.

In treating $X_{n}$ and $H_{n}$ as non-stochastic our analysis should be viewed as conditional on $X_{n}$ and $H_{n}$.

\subsection{A Brief Discussion of the Assumptions}

Among other things, Assumption 1 implies that the model is complete in that the dependent vector $y_{n}$ can be solved for in terms of $X_{n}$ and the innovation $\varepsilon_{n}$. Specifically,

$$
\begin{aligned}
& y_{n}=\left(I_{n}-\lambda W_{n}\right)^{-1}\left[X_{n} \beta+u_{n}\right] \\
& u_{n}=\left(I_{n}-\rho M_{n}\right)^{-1} \varepsilon_{n} .
\end{aligned}
$$

For a detailed discussion of the specification of the parameter space for the autoregressive parameters and normalizations of the spatial weights matrices see Kelejian and Prucha (2007b).

Assumption 2 allows the innovations to be heteroskedastic with uniformly bounded variances.

Given (4), Assumption 2 implies that $E\left(y_{n}\right)=\left(I_{n}-\lambda W_{n}\right)^{-1} X_{n} \beta$. Since under Assumptions 1 and 3 the roots of $W_{n}$ are all less than one in absolute value,

$$
E\left(y_{n}\right)=\left[I_{n}+\lambda W_{n}+\lambda^{2} W_{n}^{2}+\ldots\right] X_{n} \beta .
$$

We suggest a multi-step estimation procedure below. In the first step instruments are needed for $Z_{n}$, and in a later step instruments are needed for $M_{n} Z_{n}$. The ideal instruments are

$$
\begin{aligned}
E\left(Z_{n}\right) & =\left[X_{n}, W_{n} E\left(y_{n}\right)\right], \\
E\left(M_{n} Z_{n}\right) & =\left[M_{n} X_{n}, M_{n} W_{n} E\left(y_{n}\right)\right] .
\end{aligned}
$$


In light of (5), all of the columns of $E\left(Z_{n}\right)$ and $E\left(M_{n} Z_{n}\right)$ are linear in

$$
X_{n}, W_{n} X_{n}, W_{n}^{2} X_{n}, \ldots, M_{n} X_{n}, M_{n} W_{n} X_{n}, M_{n} W_{n}^{2} X_{n}, \ldots
$$

Let $H_{n}$ be a subset of the columns in (7), say

$$
H_{n}=\left(X_{n}, W_{n} X_{n}, \ldots, W_{n}^{q} X_{n}, M_{n} X_{n}, M_{n} W_{n} X_{n}, \ldots, M_{n} W_{n}^{q} X_{n}\right)
$$

where, typically, $q \leq 2$. Then the evident approximation to the ideal instruments for $Z_{n}$ and $M_{n} Z_{n}$ is $P_{n} Z_{n}$ and $P_{n} M_{n} Z_{n}$ where $P_{n}$ is the projection matrix: $P_{n}=H_{n}\left(H_{n}^{\prime} H_{n}\right)^{-1} H_{n}$. In passing note that, via Assumption 5, $H_{n}$ is assumed to contain at least the linearly independent columns of $X_{n}$ and $M_{n} X_{n}$, and therefore

$$
\begin{aligned}
P_{n} Z_{n} & =\left(X_{n}, P_{n} W_{n} y_{n}\right), \\
P_{n} M_{n} Z_{n} & =\left(M_{n} X_{n}, P_{n} M_{n} W_{n} y_{n}\right) .
\end{aligned}
$$

Assumption 3 is a technical assumption which is used in the large-sample derivation of the regression parameter estimator. Among other things, this assumption limits the extent of spatial autocorrelation.

Assumption 4 rules out multicollinearity problems, as well as unbounded exogenous variables. Among other things, Assumption 5 implies that there are at least as many instruments as there are regression parameters. Assumption 6 rules out redundant instruments and specifies conditions which ensure the identifiability of the regression parameter estimators.

\section{Estimators}

In this section we specify GMM and instrumental variable (IV) estimators for the model parameters $\rho$ and $\delta$. The suggested estimation procedure consists of two steps. Each step consists of substeps involving the estimation of $\rho$ and $\delta$ by GMM and IV methods. In step 1, estimates are computed from the original model (1). Those estimates are used in step 2 to compute estimates from the transformed model (3), with $\rho$ replaced by an estimator.

\subsection{Moment Conditions}

Following Kelejian and Prucha (2007b) our estimators for $\rho$ will be GMM estimators corresponding to the following population moment conditions:

$$
\begin{aligned}
& n^{-1} E \bar{\varepsilon}_{n}^{\prime} \bar{\varepsilon}_{n}=n^{-1} \operatorname{tr}\left\{M_{n}\left[\operatorname{diag}_{i=1}^{n}\left(E \varepsilon_{i, n}^{2}\right)\right] M_{n}^{\prime}\right\}, \\
& n^{-1} E \bar{\varepsilon}_{n}^{\prime} \varepsilon_{n}=0
\end{aligned}
$$

with $\bar{\varepsilon}_{n}=M_{n} \varepsilon_{n}$. Let $A_{1, n}=M_{n}^{\prime} M_{n}-\operatorname{diag}_{i=1}^{n}\left(m_{. i, n}^{\prime} m_{. i, n}\right)$ and $A_{2, n}=M_{n}$. It is readily seen that these moment conditions can also be written as.

$$
\begin{aligned}
& n^{-1} E \varepsilon_{n}^{\prime} A_{1, n} \varepsilon_{n}=n^{-1} E\left[u_{n}-\rho \bar{u}_{n}\right]^{\prime} A_{1, n}\left[u_{n}-\rho \bar{u}_{n}\right]=0, \\
& n^{-1} E \varepsilon_{n}^{\prime} A_{2, n} \varepsilon_{n}=n^{-1} E\left[u_{n}-\rho \bar{u}_{n}\right]^{\prime} A_{2, n}\left[u_{n}-\rho \bar{u}_{n}\right]=0,
\end{aligned}
$$


with $\bar{u}_{n}=M_{n} u_{n}$.

The first condition in equation (10) allows the innovations to be heteroskedastic of unknown form. If the innovations are homoskedastic with finite variance $\sigma^{2}$, this condition simplifies to

$$
n^{-1} E \bar{\varepsilon}_{n}^{\prime} \bar{\varepsilon}_{n}=\sigma^{2} n^{-1} \operatorname{tr}\left\{M_{n} M_{n}^{\prime}\right\}
$$

Under the null hypothesis of homoskedasticity, sample versions of the two conditions will converge to the same quantity.

\subsection{GMM/IV Estimators, Original Model}

\section{Step 1a: 2SLS Estimator}

In the first step, $\delta$ is estimated by 2SLS applied to model (1) using the instrument matrix $H_{n}$ in Assumption 5. Let $\widetilde{\delta}_{n}$ denote the 2SLS estimator, then

$$
\widetilde{\delta}_{n}=\left(\widetilde{Z}_{n}^{\prime} Z_{n}\right)^{-1} \widetilde{Z}_{n}^{\prime} y_{n}
$$

where $\widetilde{Z}_{n}=P_{H} Z_{n}=\left(X_{n}, \widetilde{W_{n} y_{n}}\right), \widetilde{W_{n} y_{n}}=P_{H} W_{n} y_{n}$, and where $P_{H}=H_{n}\left(H_{n}^{\prime} H_{n}\right)^{-1} H_{n}^{\prime}$. An instrument matrix such as $H_{n}$ was suggested originally in Kelejian and Prucha (1998).

Step 1b: Initial GMM Estimator of $\rho$ Based on 2SLS Residuals

In light of (1) and (12), the 2SLS residuals are $\widetilde{u}_{n}=y_{n}-Z_{n} \widetilde{\delta}_{n}$. Let $\widetilde{\bar{u}}_{n}=M_{n} \widetilde{u}_{n}$, and $\widetilde{\bar{u}}_{n}=M_{n}^{2} \widetilde{u}_{n}$. Consider the following sample moments corresponding to (11) based on estimated residuals:

$$
\begin{aligned}
m\left(\rho, \widetilde{\delta}_{n}\right) & =n^{-1}\left[\begin{array}{c}
\left(\widetilde{u}_{n}-\rho \widetilde{\bar{u}}_{n}\right)^{\prime} A_{1}\left(\widetilde{u}_{n}-\rho \widetilde{\bar{u}}_{n}\right) \\
\left(\widetilde{u}_{n}-\rho \widetilde{\bar{u}}_{n}\right)^{\prime} A_{2}\left(\widetilde{u}_{n}-\rho \overline{\bar{u}}_{n}\right)
\end{array}\right] \\
& =g_{n}\left(\widetilde{\delta}_{n}\right)-G_{n}\left(\widetilde{\delta}_{n}\right)\left[\begin{array}{c}
\rho \\
\rho^{2}
\end{array}\right]
\end{aligned}
$$

where the elements of the $2 \times 1$ vector $g_{n}$ and the $2 \times 2$ matrix $G_{n}$ are defined in Appendix B.1. Equation (13) implies that the elements of $g_{n}\left(\widetilde{\delta}_{n}\right)$ and $G_{n}\left(\widetilde{\delta}_{n}\right)$ are observable functions of $\widetilde{u}_{n}, \widetilde{\bar{u}}_{n}$, and $\widetilde{\bar{u}}_{n}$. Our initial GMM estimator for $\rho$ is defined as

$$
\check{\rho}_{n}=\underset{\rho \in\left[-a^{\rho}, a^{\rho}\right]}{\operatorname{argmin}}\left[m\left(\rho, \widetilde{\delta}_{n}\right)^{\prime} m\left(\rho, \widetilde{\delta}_{n}\right)\right]
$$

where $a^{\rho} \geq 1$. In light of the second expression in (13) the estimator can be viewed as an unweighted nonlinear least squares estimator. Given further assumptions listed below, it is consistent, but not efficient because of this lack of weighting. 
Step 1c: Efficient GMM Estimator of $\rho$ Based on 2SLS Residuals As might be anticipated from the discussion above, our efficient GMM estimator of $\rho$ is a weighted nonlinear least squares estimator. Specifically, this estimator is $\widetilde{\rho}_{n}$ where

$$
\widetilde{\rho}_{n}=\underset{\rho \in\left[-a^{\rho}, a^{\rho}\right]}{\operatorname{argmin}}\left[m\left(\rho, \widetilde{\delta}_{n}\right)^{\prime} \widetilde{\Psi}_{n}^{-1} m\left(\rho, \widetilde{\delta}_{n}\right)\right]
$$

and where the weighting matrix is $\widetilde{\Psi}_{n}^{-1}$. The matrix $\widetilde{\Psi}_{n}=\widetilde{\Psi}_{n}\left(\check{\rho}_{n}\right)$, defined in Appendix B.2, is an estimator of the variance-covariance matrix of the limiting distribution of the normalized sample moments $n^{1 / 2} m\left(\rho, \widetilde{\delta}_{n}\right)$.

\subsection{GMM/IV Estimators, Transformed Model}

\section{Step 2a: GS2SLS Estimator}

Consider the spatial Cochrane-Orcutt transformed model in (3). Analogous to Kelejian and Prucha (1998) we now define a generalized spatial two-stage least squares (GS2SLS) estimator of $\delta$ as the 2SLS estimator of the transformed model in (3) after replacing the parameter $\rho$ by $\widetilde{\rho}_{n}$ computed in Step 1c. Specifically, the GS2SLS estimator is defined as

$$
\widehat{\delta}_{n}\left(\widetilde{\rho}_{n}\right)=\left[\widehat{Z}_{n *}\left(\widetilde{\rho}_{n}\right)^{\prime} Z_{n *}\left(\widetilde{\rho}_{n}\right)\right]^{-1} \widehat{Z}_{n *}\left(\widetilde{\rho}_{n}\right)^{\prime} y_{n *}\left(\widetilde{\rho}_{n}\right)
$$

where $y_{n *}\left(\widetilde{\rho}_{n}\right)=y_{n}-\widetilde{\rho}_{n} M_{n} y_{n}, Z_{n *}\left(\widetilde{\rho}_{n}\right)=Z_{n}-\widetilde{\rho}_{n} M_{n} Z_{n}, \widehat{Z}_{n *}\left(\widetilde{\rho}_{n}\right)=P_{H} Z_{n *}\left(\widetilde{\rho}_{n}\right)$, and where $P_{H}=H_{n}\left(H_{n}^{\prime} H_{n}\right)^{-1} H_{n}^{\prime}$.

Step 2b: Efficient GMM Estimator of $\rho$ Based on GS2SLS Residuals

The GS2SLS residuals are given by $\widehat{u}_{n}=y_{n}-Z_{n} \widehat{\delta}_{n}\left(\widetilde{\rho}_{n}\right)$. Let $\widehat{\bar{u}}_{n}=M_{n} \widehat{u}_{n}$, and $\widehat{\overline{\bar{u}}}_{n}=M_{n}^{2} \widehat{u}_{n}$. Now consider the sample moments $m\left(\rho, \widehat{\delta}_{n}\right)$ obtained by replacing the 2SLS residuals in (13) by the GS2SLS residuals $\widehat{u}_{n}, \widehat{\bar{u}}_{n}$, and $\widehat{\overline{\bar{u}}}_{n}$. The efficient GMM estimator for $\rho$ based on GS2SLS residuals is now given by

$$
\widehat{\rho}_{n}=\underset{\rho \in\left[-a^{\rho}, a^{\rho}\right]}{\operatorname{argmin}}\left[m\left(\rho, \widehat{\delta}_{n}\right)^{\prime} \widehat{\Psi}_{n}^{-1} m\left(\rho, \widehat{\delta}_{n}\right)\right],
$$

where the weighting matrix is $\widehat{\Psi}_{n}^{-1}$. The matrix $\widehat{\Psi}_{n}=\widehat{\Psi}_{n}\left(\widetilde{\rho}_{n}\right)$, defined in Appendix B.3, is an estimator of the variance-covariance matrix of the limiting distribution of the normalized sample moments $n^{1 / 2} m\left(\rho, \widehat{\delta}_{n}\right){ }^{3}$

\section{Large Sample Distribution}

In this section we give results on the joint limiting distribution of the initial 2SLS estimator, $\widetilde{\delta}_{n}$, and the efficient GMM estimator of $\rho$ based on 2SLS residuals, namely $\widetilde{\rho}_{n}$. These estimators relate to the untransformed model.

\footnotetext{
${ }^{3} n^{1 / 2} m\left(\rho, \widehat{\delta}_{n}\right)$ and $n^{1 / 2} m\left(\rho, \widetilde{\delta}_{n}\right)$ have different limiting distributions.
} 
We also give the joint limiting distribution of the GS2SLS estimator $\widehat{\delta}_{n}$, and the efficient GMM estimator of $\rho$ which is based on GS2SLS residuals, namely $\widehat{\rho}_{n}$. These estimators correspond to the transformed model. Proofs are given in Appendix C.

\subsection{GMM/IV Estimators, Original Model}

In Appendix $\mathrm{C}$ we prove the following theorem concerning the join limiting distribution of $\widetilde{\rho}_{n}$ and $\widetilde{\delta}_{n}$.

Theorem 1 Suppose Assumptions 1-6 above and Assumptions A.1 and A.2 in the appendix hold. Then, $\widetilde{\rho}_{n}$ is efficient among the class of GMM estimators based on $2 S L S$ residuals, and

$$
\left[\begin{array}{c}
n^{1 / 2}\left(\widetilde{\delta}_{n}-\delta\right) \\
n^{1 / 2}\left(\widetilde{\rho}_{n}-\rho\right)
\end{array}\right] \stackrel{D}{\rightarrow} N\left(\left[0, \operatorname{plim}_{n \rightarrow \infty} \widetilde{\Omega}_{n}\left(\widetilde{\rho}_{n}\right)\right]\right.
$$

where $\operatorname{plim}_{n \rightarrow \infty} \widetilde{\Omega}_{n}\left(\widetilde{\rho}_{n}\right)$ is a positive definite matrix. For applied purposes, an expression is needed for $\widetilde{\Omega}_{n}\left(\widetilde{\rho}_{n}\right)$. This expression is given in the Appendix B.2.

The result in (18) indicates that both $\widetilde{\delta}_{n}$ and $\widetilde{\rho}_{n}$ are consistent. It also suggests that small-sample inferences concerning either $\rho, \delta$, or both can be based on the small-sample approximation

$$
\left[\begin{array}{c}
\widetilde{\delta}_{n} \\
\widetilde{\rho}_{n}
\end{array}\right] \dot{\sim} N\left(\left[\begin{array}{l}
\delta \\
\rho
\end{array}\right], n^{-1} \widetilde{\Omega}_{n}\right) .
$$

\subsection{GMM/IV Estimators, Transformed Model}

In Appendix $\mathrm{C}$ we prove the following theorem concerning the joint limiting distribution of $\widehat{\rho}_{n}$ and $\widehat{\delta}_{n}$.

Theorem 2 Suppose Assumptions 1-6 above and Assumptions A.1 and A.3 in the appendix hold. Then, $\widehat{\rho}_{n}$ is efficient among the class of GMM estimators based on GS2SLS residuals, and

$$
\left[\begin{array}{c}
n^{1 / 2}\left(\widehat{\delta}_{n}-\delta\right) \\
n^{1 / 2}\left(\widehat{\rho}_{n}-\rho\right)
\end{array}\right] \stackrel{D}{\rightarrow} N\left[0, \operatorname{plim}_{n \rightarrow \infty} \widehat{\Omega}_{n}\left(\widehat{\rho}_{n}\right)\right]
$$

where $\operatorname{plim}_{n \rightarrow \infty} \widehat{\Omega}_{n}\left(\widehat{\rho}_{n}\right)$ is a positive definite matrix. For applied purposes, an expression is needed for $\widehat{\Omega}_{n}\left(\widehat{\rho}_{n}\right)$. This expression is given in the appendix B.3.

Clearly, Theorem 2 implies that both $\widehat{\delta}_{n}$ and $\widehat{\rho}_{n}$ are consistent. It also suggests that small-sample inferences can be based on the approximation

$$
\left[\begin{array}{l}
\widehat{\delta}_{n} \\
\widehat{\rho}_{n}
\end{array}\right] \dot{\sim} N\left(\left[\begin{array}{l}
\delta \\
\rho
\end{array}\right], n^{-1} \widehat{\Omega}_{n}\right) .
$$




\section{Monte Carlo Experiments}

In this section we give Monte Carlo results which suggest that our estimators and corresponding test statistics behave well in finite samples. Our Monte Carlo model is a special case of the one specified in (1) and (2). Our experimental design is somewhat similar to those used in the literature by Kelejian and Prucha (1999, 2007a) and by Anselin and Florax (1995).

\subsection{The Model}

The model underlying our Monte Carlo experiments is a special case of the model specified in (1) and (2) with two exogenous regressors, i.e., $X_{n}=$ $\left[x_{n, 1}, x_{n, 2}\right]$ and $\beta=\left(\beta_{1}, \beta_{2}\right)^{\prime}$, and with $M_{n}=W_{n}$.

We consider two cases for the innovation vector $\varepsilon_{n}$. In one of these cases the elements of the innovation vector are i.i.d. $N\left(0, c^{2}\right)$, and so their standard deviation is $c$. In our second case the elements of the innovation vector are heteroskedastic. In this case we take the $i$-th element of the innovation vector $\varepsilon_{n}$ as

$$
\begin{aligned}
\varepsilon_{n, i} & =\sigma_{n, i} \zeta_{n, i}, \\
\sigma_{n, i} & =c \frac{d_{n, i}}{\sum_{j=1}^{n} d_{n, j} / n},
\end{aligned}
$$

where $\zeta_{n, i}$ is, for each of our considered sample sizes, i.i.d. $N(0,1)$, and $d_{n, i}$ is the number of neighbors the i-th unit has, which will be defined by the sample size, and weights matrices described below. At this point note that the average of the standard deviations of the elements of $\varepsilon_{n}$ is $c$, and thus the average standard deviation is identical to that in the homoskedastic case. Also note that these standard deviations are related to the number of neighbors each unit has. One example in which units might have different numbers of neighbors is the case in which the units differ in size. If neighbors are defined as units falling within a certain distance, then each unit in a group of smaller units could have many neighbors, while each unit in a group of larger units could have fewer neighbors. This scenario could relate to the northeastern portion of the US, as compared to western states in the US.

The parameters of the model which we will estimate are $\delta=\left(\beta_{1}, \beta_{2}, \lambda\right)^{\prime}$ and $\rho$. The specifications we use to generate 2000 repetitions for each Monte Carlo experiment are described below.

The two $n \times 1$ regressors $x_{n, 1}$ and $x_{n, 2}$ are normalized versions of income per-capita and the proportion of housing units which are rental in 1980, in 760 counties in US mid-western states. These data were taken from Kelejian and Robinson (1993). We normalized the 760 observations on these variables by subtracting from each observation the corresponding sample average, and then dividing that result by the sample standard deviation. The first $n$ values of these normalized variables were used in our Monte Carlo experiments of sample size $n$. For sample sizes larger than 760 the observations were

repeated. Finally, the same set of observations on these variables were used in all Monte Carlo repetitions. 
We considered five experimental values for $\lambda$ and for $\rho$, namely: $-.8,-.3$, 0 , .3. .8. In all of our experiments we took $\beta_{1}=\beta_{2}=1$. We consider two values for the (average) standard deviation $c$, namely: .5 and 1 .

For each approximate sample size we consider three weights matrices. ${ }^{4}$ The first is a variation of those that were considered in Kelejian and Prucha (1999, 2004). This variation is considered because it allows a convenient formulation of the innovation heteroskedasticity, which is a major focus of this paper. Specifically, this variation is an $n \times n$ matrix whose first $n / 3$ rows, except for the first row, have zeroes everywhere except for the elements in positions $(i, i+1)$ and $(i, i-1) .{ }^{5}$ In the first row, the non-zero elements are in position $(1,2)$ and $(1, n)$ so that it relates to a circular world. The non-zero elements in the first $n / 3$ rows are all $1 / 2$, e.g., these rows are row normalized because each row has 2 neighbors. The next $n / 3$ rows, say $j=$ $n / 3+1, \ldots, 2 n / 3$, have zeroes everywhere except in positions $(j, j \pm r)$, where $r=1,2, \ldots, 5$. The non-zero elements in these rows are all $1 / 10$. The last $n / 3$ rows are defined in a similar manner to the first $n / 3$ rows. Specifically, the non-zero elements in rows $j=2 n / 3+1, \ldots, n-1$ are in positions $(j, j+1)$, and $(j, j-1)$; in the last row the non-zero element are in positions $(n, 1)$ and $(n, n-1)$. The non-zero elements in these rows are all $1 / 2$. This matrix was considered for sample sizes $n=500$ and $n=1000$. We refer to this matrix below as the "circular world matrix".

The second and third sets of weights matrices are matrices which correspond to a "space" in which units located in the northeast portion of that space are smaller, closer to each other, and have more neighbors than the units corresponding to other quadrants of that space. Again, one might think of the states located in the northeastern portion of the US, as compared to western states.

To define these matrices, think of the matrix in terms of a square grid with both the $x$ and $y$ coordinates only taking on the values $1,1.5,2,2.5, \ldots \bar{m}$. Let the units in the northeast quadrant of this matrix be at the indicated discrete coordinates: $m \leq x \leq \bar{m}$ and $m \leq y \leq \bar{m}$. Let the remaining units be located only at integer values of the coordinates: $x=1,2, \ldots, m-1$ and $y=1,2, \ldots, m-1$. In this set-up it should be clear that the number of units located in the northeast quadrant is inversely related to $m$.

For this matrix we define a distance measure between any two units, $i_{1}$ and $i_{2}$, which have coordinates respectively, $\left(x_{1}, y_{1}\right)$ and $\left(x_{2}, y_{2}\right)$ as the Euclidean distance between them, namely

$$
d\left(i_{1}, i_{2}\right)=\left[\left(x_{1}-x_{2}\right)^{2}+\left(y_{1}-y_{2}\right)^{2}\right]^{1 / 2}
$$

Given this distance measure we define the $(i, j)$-th element of our row nor-

\footnotetext{
${ }^{4}$ Our discussion below will clarify this notion of "approximate" sample size.

${ }^{5}$ When $n / 3$ is not an integer, we take the smallest integer greater than $n / 3$.
} 
malized weights matrix $W$ as

$$
\begin{aligned}
& w_{i j}=w_{i j}^{*} / \sum_{j=1}^{n} w_{i j}^{*}, \\
& w_{i j}^{*}=\left\{\begin{array}{cc}
1 & \text { if } \quad 0<d\left(i_{1}, i_{2}\right) \leq 1 \\
0 & \text { else }
\end{array} .\right.
\end{aligned}
$$

For our experiments with a sample size of approximately 500, we considered two cases of this matrix namely $(m=5, \bar{m}=15)$ and $(m=14, \bar{m}=20)$. These values of $m$ and $\bar{m}$ imply sample sizes of respectively, $n=486$ and $n=485$. These values of $m$ and $\bar{m}$ were selected because they correspond to different proportions of units in the northeast quadrant, where each unit has more neighbors than units located in the other quadrants. As indicated, the number of neighbors each unit has is important because it is a determinant of the standard deviation of the innovation, see (19). In our first small-sample case, namely $(m=5, \bar{m}=15)$, approximately $25 \%$ of the units are located in the northeast quadrant; in our second case, $(m=14, \bar{m}=20)$, approximately $75 \%$ of the units are located in the northeast quadrant.

For our experiments with a sample size of approximately 1000, the two variations of this matrix we considered are $(m=7, \bar{m}=21)$ and $(m=$ $20, \bar{m}=28)$. The implied sample sizes are, respectively, $n=974$ and $n=$ 945. In these two cases, the proportion of units located in the northeast quadrant is, respectively approximately $25 \%$ and $76 \%$. Below we refer to all of these matrices as north-east modified-rook matrices. For future reference we summarize the characteristics of these four "modified rook" matrices in Table 1 below. We also illustrate a north-east modified-rook matrix, with the units indicated by the stars, in Figure 1 for the case in which $m=2$ and $\bar{m}=5$. 


\begin{tabular}{|l|l|l|l|}
\hline \multicolumn{5}{|l|}{} \\
\hline Matrix R1 & Matrix R2 & Matrix R3 & Matrix R4 \\
\hline$(m=5, \bar{m}=15)$ & $(m=7, \bar{m}=21)$ & $(m=14, \bar{m}=20)$ & $(m=20, \bar{m}=28)$ \\
\hline$n=486$ & $n=974$ & $n=485$ & $n=945$ \\
\hline$\% N E: 25 \%$ & $\% N E: 25 \%$ & $\% N E: 75 \%$ & $\% N E: 76 \%$ \\
\hline & & & \\
\hline
\end{tabular}

Figure 1: Example of a North-East Modified-Rook Matrix: $m=2$ and $\bar{m}=5$

\begin{tabular}{|l|l|l|l|l|l|l|l|l|l|}
\hline 5.0 & $*$ & & $*$ & & $*$ & $*$ & $*$ & $*$ & $*$ \\
\hline 4.5 & & & & & $*$ & $*$ & $*$ & $*$ & $*$ \\
\hline 4.0 & $*$ & & $*$ & & $*$ & $*$ & $*$ & $*$ & $*$ \\
\hline 3.5 & & & & & $*$ & $*$ & $*$ & $*$ & $*$ \\
\hline 3.0 & $*$ & & $*$ & & $*$ & $*$ & $*$ & $*$ & $*$ \\
\hline 2.5 & & & & & & & & & \\
\hline 2.0 & $*$ & & $*$ & & $*$ & & $*$ & & $*$ \\
\hline 1.5 & & & & & & & & & \\
\hline 1.0 & $*$ & & $*$ & & $*$ & & $*$ & & $*$ \\
\hline & 1.0 & 1.5 & 2.0 & 2.5 & 3.0 & 3.5 & 4.0 & 4.5 & 5.0 \\
\hline
\end{tabular}

\subsection{Monte Carlo Results}

Our Monte Carlo results are given in Tables 2-14 below. These tables contain results for the generalized spatial 2SLS estimator $\widehat{\delta}(\hat{\rho})$ defined in (16) which is based on the instrument matrix $H=\left[X, W X, W^{2} X\right]$, where $\tilde{\rho}$ is replaced by $\hat{\rho}$ which is the efficient GM estimator given in (17). Only results for the estimators of $\lambda$ and $\rho$, which are denoted in the tables as $\lambda_{G S}$ and $\rho_{G S}$, are reported. For purposes of comparison, we also report the quasi-maximum likelihood estimators of these two parameters, denoted in the tables as $\lambda_{M L}$ and $\rho_{M L}$.

The results in Tables 2-7 are from the heteroskedastic case with the larger average standard deviation in which $c=1.0$, see (19). The results in the first four of these tables are based on north-east modified-rook matrices 1-4 as described in Table 1. Tables 6 and 7 report results based on our circular world matrices, with sample sizes 500 and 1000 respectively. Tables 8-13 have the same format as Tables 2-7 except they correspond to our smaller standard deviation case $c=.5$. Finally, Table 14 reports results for the homoskedastic case in which the elements of the innovation vector are $i . i . d$. as $N\left(0, c^{2}\right), c=1$ with the north-east modified rook matrix R1. The experiments underlying Table 14 correspond to those underlying Table 2, the difference being that the former reports on the heteroskedastic case while the latter reports on the homoskedastic case. We have also performed Monte Carlo experiments under homoskedasticity corresponding to all cases considered in Tables 3-13, 
but for space reasons we have not included corresponding tables below. A full set of our results is available on our web site.

The results in Tables 2-13 are consistent with our large-sample theory, namely that $\lambda_{G S}$ and $\rho_{G S}$ are consistent estimators and, in the presence of heteroskedastic innovations, the quasi-maximum-likelihood estimators $\lambda_{M L}$ and $\rho_{M L}$ are in general not consistent. For example, notice that in all of the tables the biases of $\lambda_{G S}$ and $\rho_{G S}$ are so small that the root mean square error is approximately equal to the standard deviation. Also note that in all of the tables the rejection rates corresponding to $\lambda_{G S}$ and to $\rho_{G S}$ are quite close to the theoretical .05 level. Indeed, in each of the Tables 2-13 the average of these rejection rates over all of the experiments considered relating to both $\lambda_{G S}$ and $\rho_{G S}$ are quite close to the theoretical .05 level. For future reference we note that these averages do not mask outliers; the largest of these outliers, namely .1190 , relates $\lambda_{G S}$ in Table 4 and corresponds to the experiment $\rho=.8$ and $\lambda=.3$. In the tables there are no rejection rate outliers that relate to the estimator $\rho_{G S}$.

In contrast, the results for $\lambda_{M L}$ and $\rho_{M L}$ in the heteroskedastic cases show that the biases are typically large and, consequently, the rejection rates, especially for $\rho_{M L}$, deviate from the theoretical .05 level in many of the considered experiments. Indeed, in Tables 3-7, 9, and 11-13 the rejection rates corresponding to $\rho_{M L}$ exceed .9 in some experiments. In most of these experiments, the value of $\rho=-.8$. The rejection rates relating to $\lambda_{M L}$ are more moderate but still have outlier values ranging from $.86-1.0$ in Tables $5-7$, and 12-13. In most of these cases either $\rho$ or $\lambda$, or both are negative; actually the only exception to this is in Table 7 when $\rho=.8$ and $\lambda=0$. Interestingly, extreme rejection rates, say over .8 , for $\rho_{M L}$ and $\lambda_{M L}$ do not always occur for the same set of parameter values - see, e.g., Tables 3-5. Of course, when they do occur simultaneously either $\rho$ or $\lambda$ is negative, but typically not both.

Intuitive explanations of the table results thus far discussed as they relate to the values of $\rho$ and $\lambda$ are not straight forward. As one example, the reduced form for $y_{n}$ from the model (1) and (2) is

$$
y_{n}=\left(I_{n}-\lambda W_{n}\right)^{-1} X_{n} \beta+\left(I_{n}-\lambda W_{n}\right)^{-1}\left(I_{n}-\rho W_{n}\right)^{-1} \varepsilon_{n}
$$

If $\lambda$ is large in absolute value, say close 1.0, the variances of the elements of error vector in (20), namely $\left(I_{n}-\lambda W_{n}\right)^{-1}\left(I_{n}-\rho W_{n}\right)^{-1} \varepsilon_{n}$, will, ceteris paribus, tend to be large since $\lambda=1.0$ is a singular point of the inverse matrix. These larger variances will obviously have a negative effect on estimation precision. On the other hand, increased variation of the vector $y_{n}$ will, ceteris paribus, increase the variation in $W_{n} y_{n}$, which is a right hand side variable, and this should increase estimation precision. The net effect on estimation precision of a large value of $\lambda$ will obviously be the result of these two effects and it is not clear which of these two effects would dominate in a particular case. Similar concerns relate to the value of $\rho$ since, on the negative side, it also enters the error term in (20) in the same fashion as $\lambda$; on the positive side $\rho$ can be viewed as a regression parameter in (2) and the larger the value of $\rho$ the more $W_{n} u_{n}$ varies and so the more precision is increased! Of course, intuitive interpretations of our results are made still more complex by the 
interactive effects of $\rho$ and $\lambda$ as is evident in (20).

Returning to the tables, note from Tables 2-13 that, on average, the root mean square errors relating to $\rho_{G S}$ and to $\lambda_{G S}$ decrease as the sample size increases in every "comparable" case considered. As an example of "comparable" cases, Tables 2 and 3 both relate to a north-east modifiedrook matrix in which the north east quadrant contains $25 \%$ of the units; also the results in both of these tables are based on $c=1.0$. The main difference in the design underlying Tables 2 and 3 is the sample size, namely $n=486$ for Table 2 and $n=974$ for Table 3 . Other comparable tables are 4 and 5 , 6 and 7 , etc.

The root mean square errors for $\rho_{M L}$ and $\lambda_{M L}$ typically decrease in relevant comparisons as the sample size increases. A glance at the tables suggests that the reason for this is that the standard deviations, not the biases, decreases with the sample size. However, in all cases considered involving heteroskedasticity, described in Tables 2-13, the average root mean square errors for $\rho_{G S}$ and $\lambda_{G S}$ are less than those for $\rho_{M L}$ and $\lambda_{M L}$. For example, if in each table the ratio of the average of the root mean square error of $\rho_{G S}$ to $\rho_{M L}$ is taken, and then these ratios are averaged over the 12 tables the result is .63; this average of the root mean square errors of $\lambda_{G S}$ and $\lambda_{M L}$ is .70. Thus, over our experiments involving heteroskedasticty, the increase in efficiency of $\rho_{G S}$ relative to $\rho_{M L}$ seems to be larger than that of $\lambda_{G S}$ relative to $\lambda_{M L}$.

The "comparable" cases above focused attention on the effects of the sample size in the modified rook matrix cases by holding constant the relative size of the north-east quadrant of those matrices. We now focus attention on comparisons relating to the relative size of the north-east quadrant of those modified rook matrices by holding constant the sample size. For instance, consider the results in Tables 2 and 4. In these tables the sample sizes are, respectively 486 and 485; the proportion of units located in the north-east quadrant are, respectively, $25 \%$ and $75 \%$.

The root mean square errors for $\rho_{G S}$ and $\lambda_{G S}$ are lower in Table 4 than they are in Table 2, as are the averages of these root mean square errors. The same result holds for the root mean square errors of $\rho_{G S}$ and $\lambda_{G S}$ in Tables 3 and 5, Tables 8 and 10, and Tables 9 and 11. Thus, the larger the size of the north-east quadrant, the more precise the estimation is.

Given the complexity of our model and our estimators, there does not seem to be a simple explanation of these results. On an intuitive level, one suspects that the particular values of the instrument matrix and the variances are at least part of the explanation. For example, if $\rho=0$ the only term in the large sample distribution of $\tilde{\delta}_{n}$ that would involve the variances would be $\lim _{n \rightarrow \infty} n^{-1} H_{n}^{\prime} \Sigma_{n} H_{n}$, which clearly involves the products of the variances and the elements of $H_{n}$. On another, and simpler issue, we note that the root mean square errors are lower in Tables 8-11 than they are, respectively in 2-5. The reason for this is that $c=.5$ in Tables $8-11$, while $c=1.0$ in Tables $2-5$.

Table 14 contains results for the homoskedastic case in which the weights matrix is a north-east modified-rook matrix $\mathrm{R} 1$ and $c=1.0$. Under homoskedasticity the quasi-maximum-likelihood estimator is the maximum- 
likelihood estimator, so it is consistent and efficient. Of course, in this case both $\rho_{G S}$ and $\lambda_{G S}$ are also consistent. Consistent with this, note from Table 14 that the biases are small for all four of the indicated estimators, and the rejection rates are reasonably close to the theoretical .05 level. Although the root mean square errors are relatively small for both $\rho_{G S}$ and $\lambda_{G S}$ they are typically larger than those of $\rho_{M L}$ and $\lambda_{M L}$. On average the root mean square errors of $\rho_{G S}$ and $\lambda_{G S}$ are, respectively $5 \%$ and $8 \%$ larger than those of $\rho_{M L}$ and $\lambda_{M L}$. 
Table 2: Heteroskedasticity with c=1, Modified Rook Matrix R1 ( $\mathrm{n}=486$ )

\begin{tabular}{|c|c|c|c|c|c|c|c|c|c|}
\hline \multirow[b]{2}{*}{ rho } & \multirow[b]{2}{*}{ lambda } & \multicolumn{4}{|c|}{$\rho_{G S}$} & \multicolumn{4}{|c|}{$\rho_{M L}$} \\
\hline & & Median & Std. err & Rej. Rate & RMSE & Median & Std. err & Rej. Rate & RMSE \\
\hline-.8 & -.8 & -0.7870 & 0.1242 & 0.0425 & 0.1248 & -0.6464 & 0.0634 & 0.3000 & 0.1661 \\
\hline-.8 & -.3 & -0.7899 & 0.1167 & 0.0420 & 0.1172 & -0.5918 & 0.0659 & 0.7100 & 0.2184 \\
\hline-.8 & 0 & -0.7902 & 0.1117 & 0.0420 & 0.1121 & -0.5847 & 0.0676 & 0.7625 & 0.2256 \\
\hline-.8 & .3 & -0.7923 & 0.1081 & 0.0430 & 0.1083 & -0.5863 & 0.0675 & 0.7715 & 0.2241 \\
\hline-.8 & .8 & -0.7922 & 0.1025 & 0.0470 & 0.1028 & -0.6036 & 0.0658 & 0.7200 & 0.2071 \\
\hline-.3 & -.8 & -0.2974 & 0.1285 & 0.0480 & 0.1285 & -0.3047 & 0.0949 & 0.0190 & 0.0950 \\
\hline-.3 & -.3 & -0.2964 & 0.1344 & 0.0485 & 0.1345 & -0.2473 & 0.0907 & 0.0410 & 0.1049 \\
\hline-.3 & 0 & -0.2960 & 0.1317 & 0.0500 & 0.1317 & -0.2281 & 0.0913 & 0.0675 & 0.1162 \\
\hline-.3 & .3 & -0.2949 & 0.1279 & 0.0490 & 0.1280 & -0.2185 & 0.0936 & 0.0915 & 0.1241 \\
\hline-.3 & .8 & -0.2944 & 0.1198 & 0.0535 & 0.1199 & -0.2269 & 0.0916 & 0.0910 & 0.1172 \\
\hline 0 & -.8 & 0.0013 & 0.1190 & 0.0510 & 0.1190 & -0.0685 & 0.1023 & 0.0810 & 0.1232 \\
\hline 0 & -.3 & -0.0007 & 0.1262 & 0.0505 & 0.1262 & -0.0276 & 0.0961 & 0.0335 & 0.1000 \\
\hline 0 & 0 & -0.0011 & 0.1267 & 0.0480 & 0.1267 & -0.0072 & 0.0971 & 0.0265 & 0.0973 \\
\hline 0 & .3 & -0.0011 & 0.1254 & 0.0465 & 0.1254 & 0.0073 & 0.0977 & 0.0310 & 0.0980 \\
\hline 0 & .8 & 0.0013 & 0.1177 & 0.0510 & 0.1177 & 0.0074 & 0.0986 & 0.0380 & 0.0989 \\
\hline .3 & -.8 & 0.3019 & 0.1012 & 0.0510 & 0.1012 & 0.2013 & 0.1012 & 0.1805 & 0.1414 \\
\hline .3 & -.3 & 0.2967 & 0.1092 & 0.0560 & 0.1092 & 0.2182 & 0.0974 & 0.1190 & 0.1272 \\
\hline .3 & 0 & 0.2958 & 0.1116 & 0.0500 & 0.1117 & 0.2356 & 0.0972 & 0.0855 & 0.1166 \\
\hline .3 & .3 & 0.2947 & 0.1122 & 0.0500 & 0.1123 & 0.2529 & 0.0978 & 0.0665 & 0.1085 \\
\hline .3 & .8 & 0.2971 & 0.1076 & 0.0475 & 0.1077 & 0.2642 & 0.0980 & 0.0670 & 0.1044 \\
\hline .8 & -.8 & 0.7992 & 0.0511 & 0.0535 & 0.0512 & 0.7364 & 0.0608 & 0.2960 & 0.0880 \\
\hline .8 & -.3 & 0.7937 & 0.0572 & 0.0560 & 0.0575 & 0.7240 & 0.0659 & 0.2785 & 0.1005 \\
\hline .8 & 0 & 0.7885 & 0.0615 & 0.0695 & 0.0625 & 0.7254 & 0.0691 & 0.2210 & 0.1017 \\
\hline .8 & .3 & 0.7822 & 0.0685 & 0.0695 & 0.0708 & 0.7330 & 0.0733 & 0.1700 & 0.0993 \\
\hline .8 & .8 & 0.7814 & 0.0692 & 0.0575 & 0.0717 & 0.7525 & 0.0819 & 0.1500 & 0.0947 \\
\hline \multicolumn{2}{|c|}{ average } & 0.0000 & 0.1068 & 0.0509 & 0.1072 & 0.0207 & 0.0851 & 0.2167 & 0.1279 \\
\hline \multirow[b]{2}{*}{ rho } & \multirow[b]{2}{*}{ lambda } & \multicolumn{4}{|c|}{$\lambda_{G S}$} & \multicolumn{4}{|c|}{$\lambda_{M L}$} \\
\hline & & Median & Std. err & Rej. Rate & RMSE & \multicolumn{4}{|c|}{\begin{tabular}{l|l}
\multicolumn{3}{c}{$\lambda_{M L}$} \\
Std. err & Rej. Rate
\end{tabular}} \\
\hline-.8 & -.8 & -0.8093 & 0.0627 & 0.0525 & 0.0634 & -0.7650 & 0.0384 & 0.0145 & 0.0520 \\
\hline-.8 & -.3 & -0.3050 & 0.0612 & 0.0495 & 0.0614 & -0.3409 & 0.0494 & 0.0525 & 0.0642 \\
\hline-.8 & 0 & -0.0031 & 0.0505 & 0.0515 & 0.0506 & -0.0510 & 0.0454 & 0.1070 & 0.0683 \\
\hline-.8 & .3 & 0.2982 & 0.0373 & 0.0505 & 0.0373 & 0.2570 & 0.0357 & 0.1265 & 0.0559 \\
\hline-.8 & .8 & 0.7996 & 0.0120 & 0.0495 & 0.0120 & 0.7899 & 0.0112 & 0.0710 & 0.0151 \\
\hline-.3 & -.8 & -0.8014 & 0.0433 & 0.0380 & 0.0434 & -0.7307 & 0.0329 & 0.1220 & 0.0767 \\
\hline-.3 & -.3 & -0.3028 & 0.0574 & 0.0485 & 0.0575 & -0.2957 & 0.0476 & 0.0075 & 0.0478 \\
\hline-.3 & 0 & -0.0019 & 0.0520 & 0.0490 & 0.0520 & -0.0208 & 0.0455 & 0.0230 & 0.0500 \\
\hline-.3 & .3 & 0.2994 & 0.0410 & 0.0480 & 0.0410 & 0.2722 & 0.0376 & 0.0450 & 0.0468 \\
\hline-.3 & .8 & 0.7997 & 0.0140 & 0.0480 & 0.0140 & 0.7905 & 0.0130 & 0.0480 & 0.0161 \\
\hline 0 & -.8 & -0.8005 & 0.0367 & 0.0380 & 0.0367 & -0.7279 & 0.0300 & 0.1825 & 0.0781 \\
\hline 0 & -.3 & -0.2996 & 0.0552 & 0.0455 & 0.0552 & -0.2734 & 0.0473 & 0.0190 & 0.0542 \\
\hline 0 & 0 & 0.0004 & 0.0543 & 0.0475 & 0.0543 & -0.0020 & 0.0481 & 0.0110 & 0.0482 \\
\hline 0 & .3 & 0.3002 & 0.0455 & 0.0460 & 0.0455 & 0.2828 & 0.0419 & 0.0260 & 0.0453 \\
\hline 0 & .8 & 0.8001 & 0.0167 & 0.0505 & 0.0167 & 0.7910 & 0.0154 & 0.0405 & 0.0178 \\
\hline .3 & -.8 & -0.7991 & 0.0327 & 0.0440 & 0.0327 & -0.7307 & 0.0286 & 0.2295 & 0.0750 \\
\hline .3 & -.3 & -0.2972 & 0.0578 & 0.0455 & 0.0578 & -0.2523 & 0.0497 & 0.0550 & 0.0689 \\
\hline .3 & 0 & 0.0028 & 0.0587 & 0.0465 & 0.0588 & 0.0187 & 0.0530 & 0.0240 & 0.0562 \\
\hline .3 & .3 & 0.3027 & 0.0530 & 0.0510 & 0.0531 & 0.2959 & 0.0487 & 0.0175 & 0.0489 \\
\hline .3 & .8 & 0.8013 & 0.0216 & 0.0485 & 0.0216 & 0.7913 & 0.0204 & 0.0390 & 0.0222 \\
\hline .8 & -.8 & -0.7980 & 0.0288 & 0.0520 & 0.0288 & -0.7421 & 0.0243 & 0.2160 & 0.0627 \\
\hline .8 & -.3 & -0.2877 & 0.0659 & 0.0735 & 0.0671 & -0.2189 & 0.0570 & 0.1175 & 0.0992 \\
\hline .8 & 0 & 0.0186 & 0.0791 & 0.1000 & 0.0813 & 0.0657 & 0.0678 & 0.0820 & 0.0944 \\
\hline .8 & .3 & 0.3260 & 0.0858 & 0.1120 & 0.0897 & 0.3394 & 0.0740 & 0.0670 & 0.0838 \\
\hline .8 & .8 & 0.8158 & 0.0474 & 0.0975 & 0.0500 & 0.7962 & 0.0535 & 0.0920 & 0.0536 \\
\hline & rage & 0.0024 & 0.0468 & 0.0553 & 0.0473 & 0.0136 & 0.0407 & 0.0734 & 0.0561 \\
\hline
\end{tabular}


Table 3: Heteroskedasticity with $\mathrm{c}=1$, Modified Rook Matrix R2 ( $\mathrm{n}=974)$

\begin{tabular}{|c|c|c|c|c|c|c|c|c|c|}
\hline \multirow[b]{2}{*}{ rho } & \multirow[b]{2}{*}{ lambda } & \multicolumn{4}{|c|}{$\rho_{G S}$} & \multicolumn{4}{|c|}{$\rho_{M L}$} \\
\hline & & Median & Std. err & Rej. Rate & RMSE & Median & Std. err & Rej. Rate & RMSE \\
\hline-.8 & -.8 & -0.7963 & 0.0945 & 0.0495 & 0.0946 & -0.6485 & 0.0471 & 0.6840 & 0.1587 \\
\hline-.8 & -.3 & -0.7977 & 0.0853 & 0.0400 & 0.0853 & -0.5884 & 0.0494 & 0.9760 & 0.2173 \\
\hline-.8 & 0 & -0.7979 & 0.0819 & 0.0390 & 0.0820 & -0.5796 & 0.0501 & 0.9855 & 0.2260 \\
\hline-.8 & .3 & -0.7980 & 0.0800 & 0.0415 & 0.0800 & -0.5806 & 0.0504 & 0.9860 & 0.2251 \\
\hline-.8 & .8 & -0.7985 & 0.0762 & 0.0445 & 0.0763 & -0.6005 & 0.0492 & 0.9770 & 0.2055 \\
\hline-.3 & -.8 & -0.3003 & 0.0983 & 0.0530 & 0.0983 & -0.3132 & 0.0678 & 0.0245 & 0.0691 \\
\hline-.3 & -.3 & -0.3006 & 0.0991 & 0.0525 & 0.0991 & -0.2463 & 0.0663 & 0.0600 & 0.0854 \\
\hline-.3 & 0 & -0.3001 & 0.0968 & 0.0480 & 0.0968 & -0.2238 & 0.0671 & 0.1105 & 0.1015 \\
\hline-.3 & .3 & -0.3003 & 0.0935 & 0.0480 & 0.0935 & -0.2126 & 0.0679 & 0.1575 & 0.1107 \\
\hline-.3 & .8 & -0.3003 & 0.0872 & 0.0470 & 0.0872 & -0.2215 & 0.0670 & 0.1530 & 0.1032 \\
\hline 0 & -.8 & -0.0006 & 0.0914 & 0.0515 & 0.0914 & -0.0790 & 0.0735 & 0.1630 & 0.1079 \\
\hline 0 & -.3 & -0.0016 & 0.0952 & 0.0540 & 0.0952 & -0.0282 & 0.0705 & 0.0425 & 0.0760 \\
\hline 0 & 0 & -0.0021 & 0.0938 & 0.0510 & 0.0938 & -0.0050 & 0.0711 & 0.0315 & 0.0713 \\
\hline 0 & .3 & -0.0018 & 0.0914 & 0.0510 & 0.0914 & 0.0128 & 0.0714 & 0.0360 & 0.0726 \\
\hline 0 & .8 & -0.0009 & 0.0838 & 0.0530 & 0.0838 & 0.0151 & 0.0697 & 0.0495 & 0.0714 \\
\hline .3 & -.8 & 0.2981 & 0.0746 & 0.0540 & 0.0747 & 0.1914 & 0.0711 & 0.3455 & 0.1298 \\
\hline .3 & -.3 & 0.2959 & 0.0814 & 0.0515 & 0.0815 & 0.2163 & 0.0702 & 0.2030 & 0.1093 \\
\hline .3 & 0 & 0.2951 & 0.0856 & 0.0530 & 0.0858 & 0.2371 & 0.0709 & 0.1275 & 0.0948 \\
\hline .3 & .3 & 0.2938 & 0.0858 & 0.0530 & 0.0860 & 0.2563 & 0.0715 & 0.0765 & 0.0838 \\
\hline .3 & .8 & 0.2967 & 0.0777 & 0.0505 & 0.0778 & 0.2732 & 0.0704 & 0.0715 & 0.0753 \\
\hline .8 & -.8 & 0.7994 & 0.0356 & 0.0540 & 0.0356 & 0.7321 & 0.0428 & 0.5045 & 0.0803 \\
\hline .8 & -.3 & 0.7970 & 0.0393 & 0.0600 & 0.0394 & 0.7240 & 0.0471 & 0.4875 & 0.0894 \\
\hline .8 & 0 & 0.7938 & 0.0435 & 0.0645 & 0.0439 & 0.7274 & 0.0507 & 0.3800 & 0.0886 \\
\hline .8 & .3 & 0.7896 & 0.0496 & 0.0660 & 0.0507 & 0.7346 & 0.0547 & 0.2775 & 0.0853 \\
\hline .8 & .8 & 0.7844 & 0.0588 & 0.0660 & 0.0608 & 0.7544 & 0.0625 & 0.1640 & 0.0774 \\
\hline \multicolumn{2}{|c|}{ average } & -0.0021 & 0.0792 & 0.0518 & 0.0794 & 0.0219 & 0.0620 & 0.3230 & 0.1126 \\
\hline \multirow[b]{2}{*}{ rho } & \multirow[b]{2}{*}{ lambda } & \multicolumn{4}{|c|}{$\lambda_{G S}$} & \multicolumn{4}{|c|}{$\lambda_{M L}$} \\
\hline & & Median & Std. err & Rej. Rate & RMSE & \multicolumn{4}{|c|}{\begin{tabular}{l|l}
\multicolumn{3}{c}{$\lambda_{M L}$} \\
Std. err & Rej. Rate
\end{tabular}} \\
\hline-.8 & -.8 & -0.8025 & 0.0538 & 0.0460 & 0.0538 & -0.7595 & 0.0303 & 0.0635 & 0.0506 \\
\hline-.8 & -.3 & -0.2994 & 0.0519 & 0.0505 & 0.0519 & -0.3418 & 0.0388 & 0.1100 & 0.0570 \\
\hline-.8 & 0 & 0.0009 & 0.0438 & 0.0475 & 0.0438 & -0.0537 & 0.0367 & 0.2250 & 0.0651 \\
\hline-.8 & .3 & 0.3005 & 0.0326 & 0.0485 & 0.0326 & 0.2530 & 0.0304 & 0.2855 & 0.0560 \\
\hline-.8 & .8 & 0.8002 & 0.0111 & 0.0495 & 0.0111 & 0.7874 & 0.0106 & 0.1745 & 0.0164 \\
\hline-.3 & -.8 & -0.7992 & 0.0400 & 0.0490 & 0.0400 & -0.7162 & 0.0283 & 0.5305 & 0.0885 \\
\hline-.3 & -.3 & -0.2984 & 0.0482 & 0.0450 & 0.0483 & -0.2923 & 0.0383 & 0.0175 & 0.0391 \\
\hline-.3 & 0 & 0.0016 & 0.0434 & 0.0440 & 0.0434 & -0.0202 & 0.0379 & 0.0455 & 0.0429 \\
\hline-.3 & .3 & 0.3008 & 0.0354 & 0.0465 & 0.0354 & 0.2703 & 0.0324 & 0.0955 & 0.0440 \\
\hline-.3 & .8 & 0.8007 & 0.0135 & 0.0485 & 0.0136 & 0.7886 & 0.0127 & 0.1140 & 0.0170 \\
\hline 0 & -.8 & -0.7989 & 0.0352 & 0.0500 & 0.0352 & -0.7103 & 0.0267 & 0.6985 & 0.0936 \\
\hline 0 & -.3 & -0.2974 & 0.0489 & 0.0515 & 0.0490 & -0.2675 & 0.0395 & 0.0590 & 0.0512 \\
\hline 0 & 0 & 0.0023 & 0.0471 & 0.0505 & 0.0472 & 0.0007 & 0.0406 & 0.0225 & 0.0406 \\
\hline 0 & .3 & 0.3021 & 0.0391 & 0.0470 & 0.0391 & 0.2826 & 0.0361 & 0.0510 & 0.0400 \\
\hline 0 & .8 & 0.8011 & 0.0159 & 0.0500 & 0.0159 & 0.7894 & 0.0152 & 0.0910 & 0.0185 \\
\hline .3 & -.8 & -0.7972 & 0.0317 & 0.0560 & 0.0318 & -0.7115 & 0.0260 & 0.7645 & 0.0922 \\
\hline .3 & -.3 & -0.2956 & 0.0494 & 0.0560 & 0.0496 & -0.2422 & 0.0420 & 0.1520 & 0.0714 \\
\hline .3 & 0 & 0.0039 & 0.0517 & 0.0575 & 0.0518 & 0.0235 & 0.0442 & 0.0575 & 0.0500 \\
\hline .3 & .3 & 0.3035 & 0.0451 & 0.0550 & 0.0452 & 0.2979 & 0.0411 & 0.0330 & 0.0412 \\
\hline .3 & .8 & 0.8020 & 0.0206 & 0.0480 & 0.0207 & 0.7906 & 0.0197 & 0.0650 & 0.0218 \\
\hline .8 & -.8 & -0.7980 & 0.0277 & 0.0590 & 0.0278 & -0.7279 & 0.0205 & 0.7180 & 0.0750 \\
\hline .8 & -.3 & -0.2909 & 0.0536 & 0.0730 & 0.0543 & -0.2094 & 0.0455 & 0.3025 & 0.1013 \\
\hline .8 & 0 & 0.0158 & 0.0645 & 0.0885 & 0.0664 & 0.0697 & 0.0545 & 0.1665 & 0.0885 \\
\hline .8 & .3 & 0.3191 & 0.0688 & 0.1010 & 0.0714 & 0.3409 & 0.0584 & 0.0990 & 0.0713 \\
\hline .8 & .8 & 0.8146 & 0.0430 & 0.1030 & 0.0454 & 0.7999 & 0.0442 & 0.1010 & 0.0442 \\
\hline & rage & 0.0037 & 0.0406 & 0.0568 & 0.0410 & 0.0177 & 0.0340 & 0.2017 & 0.0551 \\
\hline
\end{tabular}


Table 4: Heteroskedasticity with c=1, Modified Rook Matrix R3 (n=485)

\begin{tabular}{|c|c|c|c|c|c|c|c|c|c|}
\hline \multirow[b]{2}{*}{ rho } & \multirow[b]{2}{*}{ lambda } & \multicolumn{4}{|c|}{$\rho_{G S}$} & \multicolumn{4}{|c|}{$\rho_{M L}$} \\
\hline & & Median & Std. err & Rej. Rate & RMSE & Median & Std. err & Rej. Rate & RMSE \\
\hline-.8 & -.8 & -0.7813 & 0.0853 & 0.0630 & 0.0873 & -0.6909 & 0.0531 & 0.2560 & 0.1213 \\
\hline-.8 & -.3 & -0.7885 & 0.0668 & 0.0500 & 0.0678 & -0.6307 & 0.0521 & 0.8755 & 0.1771 \\
\hline-.8 & 0 & -0.7918 & 0.0592 & 0.0485 & 0.0597 & -0.6264 & 0.0524 & 0.9240 & 0.1814 \\
\hline-.8 & .3 & -0.7936 & 0.0556 & 0.0485 & 0.0559 & -0.6328 & 0.0517 & 0.9240 & 0.1750 \\
\hline-.8 & .8 & -0.7935 & 0.0517 & 0.0510 & 0.0521 & -0.6552 & 0.0468 & 0.8845 & 0.1522 \\
\hline-.3 & -.8 & -0.2858 & 0.1008 & 0.0560 & 0.1018 & -0.3133 & 0.0788 & 0.0375 & 0.0799 \\
\hline-.3 & -.3 & -0.2907 & 0.1052 & 0.0590 & 0.1056 & -0.2420 & 0.0696 & 0.0815 & 0.0906 \\
\hline-.3 & 0 & -0.2919 & 0.1026 & 0.0590 & 0.1029 & -0.2163 & 0.0703 & 0.1300 & 0.1093 \\
\hline-.3 & .3 & -0.2912 & 0.0994 & 0.0580 & 0.0998 & -0.2029 & 0.0730 & 0.1860 & 0.1215 \\
\hline-.3 & .8 & -0.2899 & 0.0921 & 0.0610 & 0.0926 & -0.2138 & 0.0730 & 0.1810 & 0.1130 \\
\hline 0 & -.8 & 0.0103 & 0.1026 & 0.0570 & 0.1031 & -0.0789 & 0.0905 & 0.1490 & 0.1200 \\
\hline 0 & -.3 & 0.0070 & 0.1073 & 0.0595 & 0.1075 & -0.0350 & 0.0812 & 0.0610 & 0.0884 \\
\hline 0 & 0 & 0.0059 & 0.1087 & 0.0620 & 0.1089 & -0.0084 & 0.0815 & 0.0490 & 0.0820 \\
\hline 0 & .3 & 0.0056 & 0.1070 & 0.0610 & 0.1072 & 0.0108 & 0.0824 & 0.0600 & 0.0831 \\
\hline 0 & .8 & 0.0070 & 0.1005 & 0.0580 & 0.1007 & 0.0135 & 0.0829 & 0.0755 & 0.0840 \\
\hline .3 & -.8 & 0.3068 & 0.0960 & 0.0620 & 0.0962 & 0.1839 & 0.0969 & 0.3425 & 0.1512 \\
\hline .3 & -.3 & 0.3025 & 0.0989 & 0.0600 & 0.0989 & 0.1976 & 0.0871 & 0.2550 & 0.1344 \\
\hline .3 & 0 & 0.3025 & 0.1014 & 0.0595 & 0.1014 & 0.2182 & 0.0855 & 0.1625 & 0.1183 \\
\hline .3 & .3 & 0.3016 & 0.1032 & 0.0595 & 0.1032 & 0.2398 & 0.0858 & 0.1085 & 0.1048 \\
\hline .3 & .8 & 0.3027 & 0.0993 & 0.0580 & 0.0993 & 0.2563 & 0.0898 & 0.1165 & 0.0999 \\
\hline .8 & -.8 & 0.7994 & 0.0541 & 0.0530 & 0.0541 & 0.7207 & 0.0610 & 0.5010 & 0.1001 \\
\hline .8 & -.3 & 0.7949 & 0.0558 & 0.0595 & 0.0560 & 0.7061 & 0.0661 & 0.5225 & 0.1149 \\
\hline .8 & 0 & 0.7887 & 0.0610 & 0.0700 & 0.0621 & 0.7063 & 0.0672 & 0.4675 & 0.1153 \\
\hline .8 & .3 & 0.7828 & 0.0687 & 0.0700 & 0.0708 & 0.7130 & 0.0705 & 0.3630 & 0.1120 \\
\hline .8 & .8 & 0.7794 & 0.0693 & 0.0720 & 0.0723 & 0.7330 & 0.0836 & 0.2655 & 0.1071 \\
\hline \multicolumn{2}{|c|}{ average } & 0.0040 & 0.0861 & 0.0590 & 0.0867 & 0.0061 & 0.0733 & 0.3192 & 0.1175 \\
\hline \multirow[b]{2}{*}{ rho } & \multirow[b]{2}{*}{ lambda } & \multicolumn{4}{|c|}{$\lambda_{G S}$} & \multicolumn{4}{|c|}{$\lambda_{M L}$} \\
\hline & & Median & Std. err & Rej. Rate & RMSE & \multicolumn{4}{|c|}{\begin{tabular}{l|l}
\multicolumn{2}{c}{$\lambda_{M L}$} \\
Std. err & Rej. Rate
\end{tabular}} \\
\hline-.8 & -.8 & -0.8131 & 0.0593 & 0.0795 & 0.0607 & -0.7748 & 0.0365 & 0.0140 & 0.0443 \\
\hline-.8 & -.3 & -0.3065 & 0.0710 & 0.0640 & 0.0713 & -0.3591 & 0.0517 & 0.1480 & 0.0785 \\
\hline-.8 & 0 & -0.0024 & 0.0605 & 0.0535 & 0.0606 & -0.0756 & 0.0510 & 0.2875 & 0.0912 \\
\hline-.8 & .3 & 0.2994 & 0.0459 & 0.0535 & 0.0459 & 0.2350 & 0.0426 & 0.3420 & 0.0777 \\
\hline-.8 & .8 & 0.8001 & 0.0153 & 0.0560 & 0.0153 & 0.7847 & 0.0139 & 0.1975 & 0.0207 \\
\hline-.3 & -.8 & -0.8019 & 0.0309 & 0.0455 & 0.0310 & -0.7364 & 0.0291 & 0.2970 & 0.0699 \\
\hline-.3 & -.3 & -0.3024 & 0.0537 & 0.0510 & 0.0538 & -0.2926 & 0.0416 & 0.0105 & 0.0422 \\
\hline-.3 & 0 & -0.0004 & 0.0538 & 0.0485 & 0.0538 & -0.0282 & 0.0438 & 0.0490 & 0.0521 \\
\hline-.3 & .3 & 0.3006 & 0.0452 & 0.0530 & 0.0452 & 0.2583 & 0.0405 & 0.1250 & 0.0581 \\
\hline-.3 & .8 & 0.8005 & 0.0168 & 0.0535 & 0.0168 & 0.7847 & 0.0159 & 0.1565 & 0.0221 \\
\hline 0 & -.8 & -0.8003 & 0.0255 & 0.0430 & 0.0255 & -0.7375 & 0.0255 & 0.4520 & 0.0675 \\
\hline 0 & -.3 & -0.2997 & 0.0477 & 0.0525 & 0.0477 & -0.2655 & 0.0392 & 0.0395 & 0.0522 \\
\hline 0 & 0 & 0.0001 & 0.0507 & 0.0535 & 0.0507 & -0.0007 & 0.0426 & 0.0200 & 0.0426 \\
\hline 0 & .3 & 0.3008 & 0.0454 & 0.0540 & 0.0454 & 0.2759 & 0.0399 & 0.0555 & 0.0466 \\
\hline 0 & .8 & 0.8009 & 0.0191 & 0.0535 & 0.0192 & 0.7853 & 0.0181 & 0.1275 & 0.0233 \\
\hline .3 & -.8 & -0.7999 & 0.0228 & 0.0495 & 0.0228 & -0.7424 & 0.0230 & 0.5150 & 0.0620 \\
\hline .3 & -.3 & -0.2970 & 0.0476 & 0.0540 & 0.0477 & -0.2431 & 0.0411 & 0.1240 & 0.0701 \\
\hline .3 & 0 & 0.0041 & 0.0535 & 0.0555 & 0.0536 & 0.0257 & 0.0454 & 0.0375 & 0.0521 \\
\hline .3 & .3 & 0.3036 & 0.0498 & 0.0565 & 0.0499 & 0.2950 & 0.0449 & 0.0345 & 0.0451 \\
\hline .3 & .8 & 0.8013 & 0.0235 & 0.0545 & 0.0235 & 0.7868 & 0.0230 & 0.0945 & 0.0265 \\
\hline .8 & -.8 & -0.7993 & 0.0228 & 0.0535 & 0.0229 & -0.7542 & 0.0201 & 0.4745 & 0.0500 \\
\hline .8 & -.3 & -0.2925 & 0.0542 & 0.0730 & 0.0547 & -0.2156 & 0.0484 & 0.2940 & 0.0973 \\
\hline .8 & 0 & 0.0153 & 0.0711 & 0.0910 & 0.0728 & 0.0717 & 0.0590 & 0.1730 & 0.0928 \\
\hline .8 & .3 & 0.3237 & 0.0816 & 0.1190 & 0.0850 & 0.3436 & 0.0660 & 0.1030 & 0.0791 \\
\hline .8 & .8 & 0.8180 & 0.0476 & 0.1100 & 0.0509 & 0.7982 & 0.0547 & 0.1515 & 0.0547 \\
\hline \multicolumn{2}{|c|}{ average } & 0.0021 & 0.0446 & 0.0612 & 0.0451 & 0.0088 & 0.0383 & 0.1729 & 0.0568 \\
\hline
\end{tabular}


Table 5: Heteroskedasticity with $\mathrm{c}=1$, Modified Rook Matrix R4 $(\mathrm{n}=945)$

\begin{tabular}{|c|c|c|c|c|c|c|c|c|c|}
\hline \multirow[b]{2}{*}{ rho } & \multirow[b]{2}{*}{ lambda } & \multicolumn{4}{|c|}{$\rho_{G S}$} & \multicolumn{4}{|c|}{$\rho_{M L}$} \\
\hline & & Median & Std. err & Rej. Rate & RMSE & Median & Std. err & Rej. Rate & RMSE \\
\hline-.8 & -.8 & -0.7895 & 0.0639 & 0.0525 & 0.0647 & -0.6909 & 0.0393 & 0.5945 & 0.1160 \\
\hline-.8 & -.3 & -0.7982 & 0.0495 & 0.0400 & 0.0495 & -0.6243 & 0.0390 & 0.9990 & 0.1799 \\
\hline-.8 & 0 & -0.7994 & 0.0442 & 0.0415 & 0.0442 & -0.6187 & 0.0397 & 1.0000 & 0.1856 \\
\hline-.8 & .3 & -0.7993 & 0.0400 & 0.0425 & 0.0400 & -0.6250 & 0.0380 & 1.0000 & 0.1791 \\
\hline-.8 & .8 & -0.7996 & 0.0376 & 0.0470 & 0.0376 & -0.6488 & 0.0343 & 0.9975 & 0.1551 \\
\hline-.3 & -.8 & -0.2930 & 0.0778 & 0.0470 & 0.0781 & -0.3106 & 0.0593 & 0.0330 & 0.0602 \\
\hline-.3 & -.3 & -0.2958 & 0.0770 & 0.0490 & 0.0771 & -0.2370 & 0.0518 & 0.1320 & 0.0816 \\
\hline-.3 & 0 & -0.2972 & 0.0765 & 0.0520 & 0.0765 & -0.2065 & 0.0522 & 0.2940 & 0.1071 \\
\hline-.3 & .3 & -0.2969 & 0.0743 & 0.0475 & 0.0744 & -0.1899 & 0.0530 & 0.4330 & 0.1222 \\
\hline-.3 & .8 & -0.2967 & 0.0672 & 0.0505 & 0.0673 & -0.2001 & 0.0541 & 0.4100 & 0.1136 \\
\hline 0 & -.8 & 0.0068 & 0.0769 & 0.0520 & 0.0772 & -0.0779 & 0.0675 & 0.2420 & 0.1031 \\
\hline 0 & -.3 & 0.0050 & 0.0824 & 0.0505 & 0.0825 & -0.0318 & 0.0592 & 0.0625 & 0.0672 \\
\hline 0 & 0 & 0.0026 & 0.0800 & 0.0545 & 0.0801 & -0.0006 & 0.0581 & 0.0380 & 0.0581 \\
\hline 0 & .3 & 0.0033 & 0.0802 & 0.0555 & 0.0803 & 0.0240 & 0.0593 & 0.0545 & 0.0640 \\
\hline 0 & .8 & 0.0040 & 0.0747 & 0.0520 & 0.0748 & 0.0302 & 0.0621 & 0.0795 & 0.0690 \\
\hline .3 & -.8 & 0.3064 & 0.0708 & 0.0485 & 0.0711 & 0.1836 & 0.0690 & 0.5340 & 0.1353 \\
\hline .3 & -.3 & 0.3033 & 0.0758 & 0.0510 & 0.0759 & 0.1974 & 0.0638 & 0.4130 & 0.1208 \\
\hline .3 & 0 & 0.3020 & 0.0788 & 0.0485 & 0.0789 & 0.2220 & 0.0620 & 0.2635 & 0.0996 \\
\hline .3 & .3 & 0.3011 & 0.0789 & 0.0505 & 0.0789 & 0.2484 & 0.0620 & 0.1350 & 0.0807 \\
\hline .3 & .8 & 0.3034 & 0.0736 & 0.0525 & 0.0737 & 0.2721 & 0.0656 & 0.0990 & 0.0713 \\
\hline .8 & -.8 & 0.8019 & 0.0389 & 0.0460 & 0.0389 & 0.7198 & 0.0462 & 0.7115 & 0.0925 \\
\hline .8 & -.3 & 0.7999 & 0.0402 & 0.0435 & 0.0402 & 0.7036 & 0.0493 & 0.7560 & 0.1083 \\
\hline .8 & 0 & 0.7979 & 0.0423 & 0.0485 & 0.0423 & 0.7053 & 0.0500 & 0.6895 & 0.1071 \\
\hline .8 & .3 & 0.7951 & 0.0469 & 0.0535 & 0.0472 & 0.7147 & 0.0509 & 0.5570 & 0.0994 \\
\hline .8 & .8 & 0.7892 & 0.0548 & 0.0545 & 0.0559 & 0.7377 & 0.0612 & 0.2700 & 0.0873 \\
\hline \multicolumn{2}{|c|}{ average } & 0.0023 & 0.0641 & 0.0492 & 0.0643 & 0.0119 & 0.0539 & 0.4319 & 0.1066 \\
\hline \multirow[b]{2}{*}{ rho } & \multirow[b]{2}{*}{ lambda } & \multicolumn{4}{|c|}{$\lambda_{G S}$} & \multicolumn{4}{|c|}{$\lambda_{M L}$} \\
\hline & & Median & Std. err & Rej. Rate & RMSE & \multicolumn{4}{|c|}{\begin{tabular}{l|l}
\multicolumn{2}{c}{$\lambda_{M L}$} \\
Std. err & Rej. Rate
\end{tabular}} \\
\hline-.8 & -.8 & -0.8104 & 0.0452 & 0.0740 & 0.0464 & -0.7735 & 0.0279 & 0.0330 & 0.0385 \\
\hline-.8 & -.3 & -0.3040 & 0.0556 & 0.0535 & 0.0557 & -0.3621 & 0.0387 & 0.2695 & 0.0732 \\
\hline-.8 & 0 & -0.0017 & 0.0488 & 0.0525 & 0.0488 & -0.0825 & 0.0390 & 0.5250 & 0.0912 \\
\hline-.8 & .3 & 0.2997 & 0.0372 & 0.0515 & 0.0372 & 0.2270 & 0.0332 & 0.6190 & 0.0802 \\
\hline-.8 & .8 & 0.8004 & 0.0130 & 0.0515 & 0.0130 & 0.7812 & 0.0120 & 0.4055 & 0.0223 \\
\hline-.3 & -.8 & -0.8020 & 0.0221 & 0.0550 & 0.0222 & -0.7334 & 0.0218 & 0.7245 & 0.0701 \\
\hline-.3 & -.3 & -0.3016 & 0.0405 & 0.0505 & 0.0405 & -0.2898 & 0.0300 & 0.0155 & 0.0317 \\
\hline-.3 & 0 & -0.0016 & 0.0412 & 0.0465 & 0.0412 & -0.0297 & 0.0319 & 0.0770 & 0.0436 \\
\hline-.3 & .3 & 0.2994 & 0.0352 & 0.0495 & 0.0352 & 0.2517 & 0.0296 & 0.2605 & 0.0567 \\
\hline-.3 & .8 & 0.8003 & 0.0141 & 0.0505 & 0.0141 & 0.7802 & 0.0137 & 0.3240 & 0.0241 \\
\hline 0 & -.8 & -0.8010 & 0.0184 & 0.0535 & 0.0185 & -0.7350 & 0.0185 & 0.8600 & 0.0675 \\
\hline 0 & -.3 & -0.3009 & 0.0359 & 0.0505 & 0.0359 & -0.2609 & 0.0288 & 0.0815 & 0.0485 \\
\hline 0 & 0 & -0.0004 & 0.0394 & 0.0495 & 0.0394 & -0.0013 & 0.0314 & 0.0155 & 0.0314 \\
\hline 0 & .3 & 0.2996 & 0.0357 & 0.0440 & 0.0357 & 0.2705 & 0.0303 & 0.0850 & 0.0423 \\
\hline 0 & .8 & 0.8004 & 0.0161 & 0.0490 & 0.0161 & 0.7808 & 0.0157 & 0.2290 & 0.0247 \\
\hline .3 & -.8 & -0.8005 & 0.0172 & 0.0535 & 0.0172 & -0.7406 & 0.0165 & 0.8870 & 0.0617 \\
\hline .3 & -.3 & -0.3006 & 0.0360 & 0.0590 & 0.0361 & -0.2365 & 0.0298 & 0.3075 & 0.0701 \\
\hline .3 & 0 & 0.0001 & 0.0394 & 0.0495 & 0.0394 & 0.0280 & 0.0331 & 0.0515 & 0.0434 \\
\hline .3 & .3 & 0.3005 & 0.0391 & 0.0480 & 0.0391 & 0.2930 & 0.0335 & 0.0205 & 0.0342 \\
\hline .3 & .8 & 0.8008 & 0.0195 & 0.0470 & 0.0196 & 0.7828 & 0.0198 & 0.1350 & 0.0262 \\
\hline .8 & -.8 & -0.8009 & 0.0162 & 0.0555 & 0.0162 & -0.7549 & 0.0150 & 0.8085 & 0.0476 \\
\hline .8 & -.3 & -0.2988 & 0.0389 & 0.0545 & 0.0389 & -0.2119 & 0.0354 & 0.5825 & 0.0950 \\
\hline .8 & 0 & 0.0051 & 0.0497 & 0.0625 & 0.0499 & 0.0746 & 0.0432 & 0.3110 & 0.0862 \\
\hline .8 & .3 & 0.3101 & 0.0556 & 0.0760 & 0.0565 & 0.3446 & 0.0462 & 0.1120 & 0.0642 \\
\hline .8 & .8 & 0.8093 & 0.0386 & 0.0790 & 0.0397 & 0.7956 & 0.0418 & 0.0945 & 0.0421 \\
\hline \multicolumn{2}{|c|}{ average } & 0.0001 & 0.0339 & 0.0546 & 0.0341 & 0.0079 & 0.0287 & 0.3134 & 0.0527 \\
\hline
\end{tabular}


Table 6: Heteroskedasticity with $\mathrm{c}=1$, Circular Matrix $(\mathrm{n}=500)$

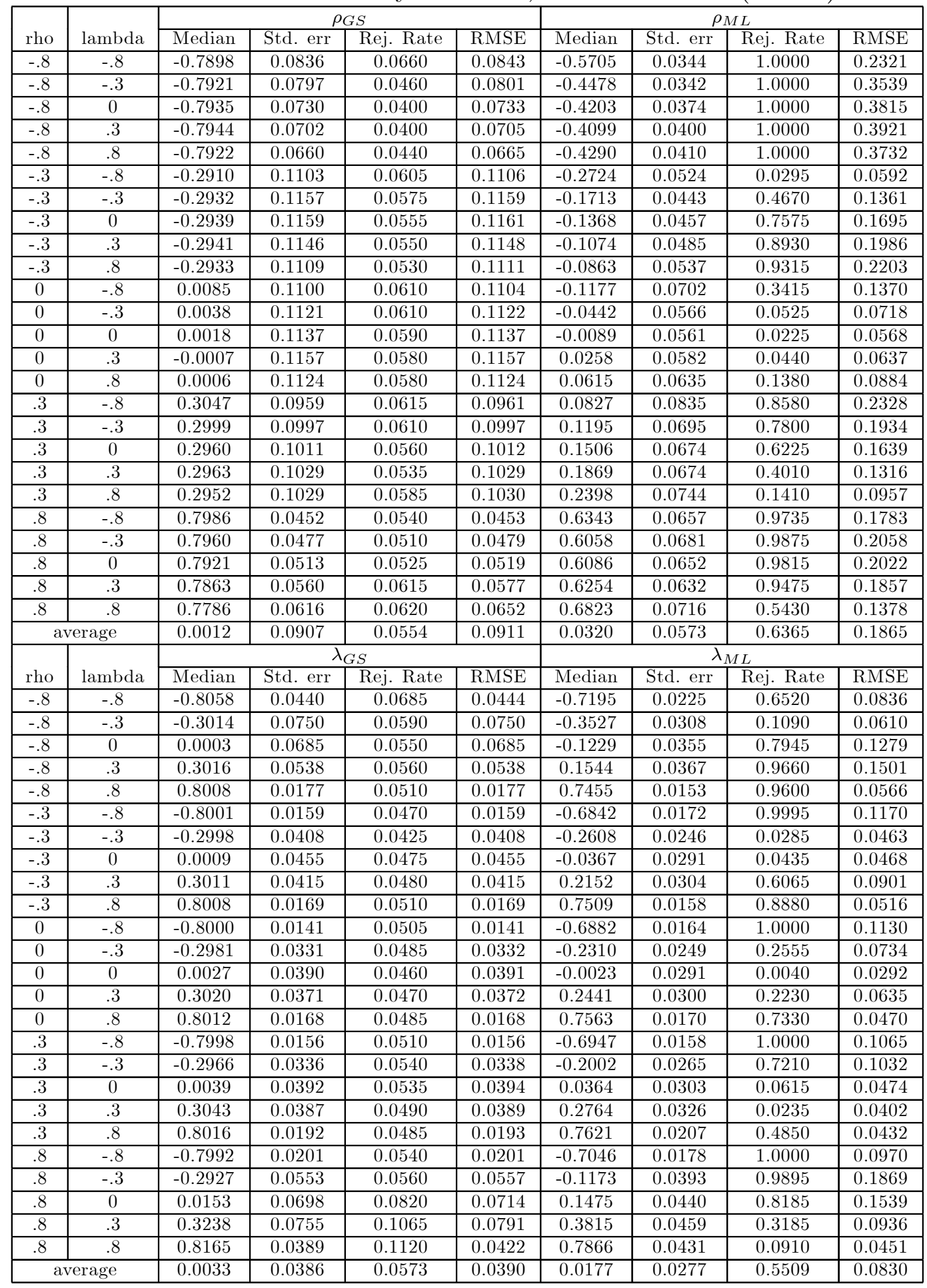


Table 7: Heteroskedasticity with $\mathrm{c}=1$, Circular Matrix $(\mathrm{n}=1000)$

\begin{tabular}{|c|c|c|c|c|c|c|c|c|c|}
\hline \multirow[b]{2}{*}{ rho } & \multirow[b]{2}{*}{ lambda } & \multicolumn{4}{|c|}{$\rho_{G S}$} & \multicolumn{4}{|c|}{$\rho_{M L}$} \\
\hline & & Median & Std. err & Rej. Rate & RMSE & Median & Std. err & Rej. Rate & RMSE \\
\hline-.8 & -.8 & -0.7926 & 0.0599 & 0.0490 & 0.0604 & -0.5718 & 0.0244 & 1.0000 & 0.2295 \\
\hline-.8 & -.3 & -0.7947 & 0.0571 & 0.0360 & 0.0573 & -0.4447 & 0.0254 & 1.0000 & 0.3562 \\
\hline-.8 & 0 & -0.7953 & 0.0532 & 0.0360 & 0.0534 & -0.4151 & 0.0278 & 1.0000 & 0.3859 \\
\hline-.8 & .3 & -0.7960 & 0.0507 & 0.0375 & 0.0509 & -0.4025 & 0.0304 & 1.0000 & 0.3987 \\
\hline-.8 & .8 & -0.7953 & 0.0474 & 0.0395 & 0.0476 & -0.4226 & 0.0315 & 1.0000 & 0.3787 \\
\hline-.3 & -.8 & -0.2919 & 0.0821 & 0.0570 & 0.0825 & -0.2689 & 0.0383 & 0.0405 & 0.0493 \\
\hline-.3 & -.3 & -0.2918 & 0.0843 & 0.0555 & 0.0847 & -0.1656 & 0.0333 & 0.8980 & 0.1385 \\
\hline-.3 & 0 & -0.2920 & 0.0863 & 0.0555 & 0.0867 & -0.1289 & 0.0348 & 0.9910 & 0.1746 \\
\hline-.3 & .3 & -0.2936 & 0.0865 & 0.0540 & 0.0867 & -0.0961 & 0.0371 & 0.9995 & 0.2072 \\
\hline-.3 & .8 & -0.2937 & 0.0830 & 0.0525 & 0.0833 & -0.0708 & 0.0419 & 1.0000 & 0.2330 \\
\hline 0 & -.8 & 0.0088 & 0.0825 & 0.0560 & 0.0830 & -0.1141 & 0.0509 & 0.6005 & 0.1250 \\
\hline 0 & -.3 & 0.0068 & 0.0843 & 0.0550 & 0.0846 & -0.0385 & 0.0418 & 0.0790 & 0.0568 \\
\hline 0 & 0 & 0.0062 & 0.0848 & 0.0545 & 0.0850 & -0.0016 & 0.0422 & 0.0230 & 0.0423 \\
\hline 0 & .3 & 0.0054 & 0.0861 & 0.0530 & 0.0863 & 0.0363 & 0.0437 & 0.0950 & 0.0568 \\
\hline 0 & .8 & 0.0060 & 0.0837 & 0.0535 & 0.0839 & 0.0788 & 0.0493 & 0.3340 & 0.0929 \\
\hline .3 & -.8 & 0.3055 & 0.0715 & 0.0545 & 0.0717 & 0.0872 & 0.0620 & 0.9845 & 0.2217 \\
\hline .3 & -.3 & 0.3036 & 0.0746 & 0.0550 & 0.0747 & 0.1252 & 0.0516 & 0.9630 & 0.1822 \\
\hline .3 & 0 & 0.3031 & 0.0762 & 0.0575 & 0.0763 & 0.1577 & 0.0495 & 0.8630 & 0.1506 \\
\hline .3 & .3 & 0.3022 & 0.0782 & 0.0565 & 0.0782 & 0.1968 & 0.0498 & 0.6045 & 0.1146 \\
\hline .3 & .8 & 0.3029 & 0.0762 & 0.0555 & 0.0763 & 0.2574 & 0.0563 & 0.1645 & 0.0706 \\
\hline .8 & -.8 & 0.8007 & 0.0328 & 0.0560 & 0.0328 & 0.6361 & 0.0485 & 1.0000 & 0.1709 \\
\hline .8 & -.3 & 0.7996 & 0.0350 & 0.0560 & 0.0350 & 0.6049 & 0.0499 & 1.0000 & 0.2014 \\
\hline .8 & 0 & 0.7974 & 0.0372 & 0.0585 & 0.0373 & 0.6074 & 0.0485 & 1.0000 & 0.1986 \\
\hline .8 & .3 & 0.7938 & 0.0422 & 0.0600 & 0.0426 & 0.6261 & 0.0470 & 0.9965 & 0.1802 \\
\hline .8 & .8 & 0.7877 & 0.0493 & 0.0630 & 0.0508 & 0.6906 & 0.0572 & 0.7100 & 0.1235 \\
\hline \multicolumn{2}{|c|}{ average } & 0.0037 & 0.0674 & 0.0527 & 0.0677 & 0.0385 & 0.0429 & 0.7339 & 0.1816 \\
\hline \multirow[b]{2}{*}{ rho } & \multirow[b]{2}{*}{ lambda } & \multicolumn{4}{|c|}{$\lambda_{G S}$} & \multicolumn{4}{|c|}{$\lambda_{M L}$} \\
\hline & & \multicolumn{3}{|c|}{ Std err I Rej Rate } & RMSE & Median & Std. err & Rej. Rate & RMSE \\
\hline-.8 & -.8 & -0.8036 & 0.0337 & 0.0760 & 0.0339 & -0.7188 & 0.0165 & 0.9835 & 0.0828 \\
\hline-.8 & -.3 & -0.3024 & 0.0556 & 0.0625 & 0.0556 & -0.3543 & 0.0227 & 0.3115 & 0.0588 \\
\hline-.8 & 0 & -0.0014 & 0.0506 & 0.0600 & 0.0506 & -0.1273 & 0.0268 & 0.9880 & 0.1301 \\
\hline-.8 & .3 & 0.2994 & 0.0399 & 0.0575 & 0.0399 & 0.1482 & 0.0266 & 1.0000 & 0.1541 \\
\hline-.8 & .8 & 0.7997 & 0.0131 & 0.0555 & 0.0131 & 0.7428 & 0.0120 & 0.9995 & 0.0585 \\
\hline-.3 & -.8 & -0.8011 & 0.0129 & 0.0565 & 0.0129 & -0.6790 & 0.0134 & 1.0000 & 0.1217 \\
\hline-.3 & -.3 & -0.3002 & 0.0313 & 0.0550 & 0.0313 & -0.2591 & 0.0188 & 0.1320 & 0.0450 \\
\hline-.3 & 0 & -0.0004 & 0.0353 & 0.0580 & 0.0353 & -0.0376 & 0.0224 & 0.1390 & 0.0438 \\
\hline-.3 & .3 & 0.3002 & 0.0318 & 0.0555 & 0.0318 & 0.2109 & 0.0230 & 0.9280 & 0.0920 \\
\hline-.3 & .8 & 0.8001 & 0.0128 & 0.0545 & 0.0128 & 0.7471 & 0.0127 & 0.9935 & 0.0544 \\
\hline 0 & -.8 & -0.8002 & 0.0110 & 0.0580 & 0.0110 & -0.6818 & 0.0130 & 1.0000 & 0.1189 \\
\hline 0 & -.3 & -0.3000 & 0.0271 & 0.0600 & 0.0271 & -0.2269 & 0.0192 & 0.7380 & 0.0756 \\
\hline 0 & 0 & 0.0010 & 0.0311 & 0.0565 & 0.0312 & -0.0013 & 0.0225 & 0.0080 & 0.0226 \\
\hline 0 & .3 & 0.3012 & 0.0304 & 0.0525 & 0.0305 & 0.2405 & 0.0239 & 0.5370 & 0.0641 \\
\hline 0 & .8 & 0.8007 & 0.0138 & 0.0525 & 0.0138 & 0.7519 & 0.0145 & 0.9575 & 0.0502 \\
\hline .3 & -.8 & -0.7997 & 0.0121 & 0.0610 & 0.0121 & -0.6878 & 0.0132 & 1.0000 & 0.1130 \\
\hline .3 & -.3 & -0.2989 & 0.0275 & 0.0615 & 0.0275 & -0.1929 & 0.0208 & 0.9850 & 0.1091 \\
\hline .3 & 0 & 0.0012 & 0.0323 & 0.0640 & 0.0324 & 0.0401 & 0.0249 & 0.1760 & 0.0471 \\
\hline .3 & .3 & 0.3019 & 0.0328 & 0.0640 & 0.0329 & 0.2761 & 0.0267 & 0.0785 & 0.0359 \\
\hline .3 & .8 & 0.8013 & 0.0163 & 0.0550 & 0.0163 & 0.7577 & 0.0183 & 0.7890 & 0.0461 \\
\hline .8 & -.8 & -0.7989 & 0.0150 & 0.0555 & 0.0151 & -0.6961 & 0.0135 & 1.0000 & 0.1048 \\
\hline .8 & -.3 & -0.2938 & 0.0389 & 0.0650 & 0.0394 & -0.1027 & 0.0298 & 1.0000 & 0.1996 \\
\hline .8 & 0 & 0.0102 & 0.0504 & 0.0795 & 0.0514 & 0.1616 & 0.0347 & 0.9915 & 0.1653 \\
\hline .8 & .3 & 0.3151 & 0.0589 & 0.0925 & 0.0608 & 0.3909 & 0.0376 & 0.6330 & 0.0984 \\
\hline .8 & .8 & 0.8101 & 0.0342 & 0.0975 & 0.0357 & 0.7856 & 0.0366 & 0.1440 & 0.0394 \\
\hline \multicolumn{2}{|c|}{ average } & 0.0017 & 0.0300 & 0.0626 & 0.0302 & 0.0195 & 0.0218 & 0.7005 & 0.0852 \\
\hline
\end{tabular}


Table 8: Heteroskedasticity with $\mathrm{c}=.5$, Modified Rook Matrix R1 $(\mathrm{n}=486)$

\begin{tabular}{|c|c|c|c|c|c|c|c|c|c|}
\hline \multirow[b]{2}{*}{ rho } & \multirow[b]{2}{*}{ lambda } & \multicolumn{4}{|c|}{$\rho_{G S}$} & \multicolumn{4}{|c|}{$\rho_{M L}$} \\
\hline & & Median & Std. err & Rej. Rate & RMSE & Median & Std. err & Rej. Rate & RMSE \\
\hline-.8 & -.8 & -0.7928 & 0.1067 & 0.0430 & 0.1069 & -0.6260 & 0.0664 & 0.5350 & 0.1862 \\
\hline-.8 & -.3 & -0.7921 & 0.1046 & 0.0455 & 0.1049 & -0.6077 & 0.0653 & 0.6865 & 0.2031 \\
\hline-.8 & 0 & -0.7918 & 0.1033 & 0.0475 & 0.1037 & -0.6060 & 0.0652 & 0.7075 & 0.2046 \\
\hline-.8 & .3 & -0.7923 & 0.1028 & 0.0475 & 0.1031 & -0.6063 & 0.0651 & 0.7095 & 0.2043 \\
\hline-.8 & .8 & -0.7920 & 0.1006 & 0.0460 & 0.1009 & -0.6111 & 0.0626 & 0.6885 & 0.1990 \\
\hline-.3 & -.8 & -0.2940 & 0.1212 & 0.0520 & 0.1214 & -0.2601 & 0.0914 & 0.0475 & 0.0997 \\
\hline-.3 & -.3 & -0.2944 & 0.1214 & 0.0520 & 0.1216 & -0.2421 & 0.0895 & 0.0650 & 0.1067 \\
\hline-.3 & 0 & -0.2936 & 0.1206 & 0.0535 & 0.1207 & -0.2360 & 0.0903 & 0.0745 & 0.1107 \\
\hline-.3 & .3 & -0.2939 & 0.1207 & 0.0530 & 0.1209 & -0.2344 & 0.0903 & 0.0800 & 0.1116 \\
\hline-.3 & .8 & -0.2942 & 0.1185 & 0.0515 & 0.1186 & -0.2376 & 0.0902 & 0.0760 & 0.1097 \\
\hline 0 & -.8 & 0.0047 & 0.1150 & 0.0510 & 0.1151 & -0.0248 & 0.0980 & 0.0515 & 0.1010 \\
\hline 0 & -.3 & 0.0016 & 0.1177 & 0.0510 & 0.1178 & -0.0125 & 0.0961 & 0.0370 & 0.0969 \\
\hline 0 & 0 & 0.0014 & 0.1177 & 0.0515 & 0.1177 & -0.0070 & 0.0957 & 0.0375 & 0.0960 \\
\hline 0 & .3 & 0.0010 & 0.1164 & 0.0500 & 0.1164 & -0.0033 & 0.0963 & 0.0365 & 0.0964 \\
\hline 0 & .8 & 0.0043 & 0.1130 & 0.0530 & 0.1131 & -0.0038 & 0.0953 & 0.0410 & 0.0953 \\
\hline .3 & -.8 & 0.3027 & 0.1004 & 0.0530 & 0.1005 & 0.2397 & 0.0948 & 0.1180 & 0.1124 \\
\hline .3 & -.3 & 0.2998 & 0.1017 & 0.0505 & 0.1017 & 0.2420 & 0.0936 & 0.1030 & 0.1101 \\
\hline .3 & 0 & 0.2999 & 0.1024 & 0.0495 & 0.1024 & 0.2467 & 0.0941 & 0.0955 & 0.1082 \\
\hline .3 & .3 & 0.2998 & 0.1021 & 0.0505 & 0.1021 & 0.2516 & 0.0937 & 0.0880 & 0.1055 \\
\hline .3 & .8 & 0.3000 & 0.1014 & 0.0525 & 0.1014 & 0.2533 & 0.0928 & 0.0905 & 0.1039 \\
\hline .8 & -.8 & 0.7991 & 0.0497 & 0.0545 & 0.0497 & 0.7494 & 0.0562 & 0.2285 & 0.0756 \\
\hline .8 & -.3 & 0.7977 & 0.0522 & 0.0540 & 0.0522 & 0.7462 & 0.0590 & 0.2350 & 0.0799 \\
\hline .8 & 0 & 0.7957 & 0.0537 & 0.0555 & 0.0539 & 0.7453 & 0.0594 & 0.2150 & 0.0808 \\
\hline .8 & .3 & 0.7938 & 0.0562 & 0.0535 & 0.0566 & 0.7461 & 0.0613 & 0.1995 & 0.0816 \\
\hline .8 & .8 & 0.7934 & 0.0590 & 0.0535 & 0.0594 & 0.7497 & 0.0641 & 0.1915 & 0.0814 \\
\hline \multicolumn{2}{|c|}{ average } & 0.0026 & 0.0992 & 0.0510 & 0.0993 & 0.0261 & 0.0811 & 0.2175 & 0.1184 \\
\hline \multirow[b]{2}{*}{ rho } & \multirow[b]{2}{*}{ lambda } & & \multicolumn{4}{|c|}{$\lambda_{M L}$} \\
\hline & & \multicolumn{4}{|c|}{\begin{tabular}{c|c}
\multicolumn{2}{c}{$\lambda_{G S}$} \\
Std. err Rej. Rate
\end{tabular}} & Median & Std. err & Rej. Rate & RMSE \\
\hline-.8 & -.8 & -0.8031 & 0.0318 & 0.0470 & 0.0319 & -0.7878 & 0.0247 & 0.0110 & 0.0276 \\
\hline-.8 & -.3 & -0.3018 & 0.0305 & 0.0480 & 0.0305 & -0.3125 & 0.0283 & 0.0320 & 0.0310 \\
\hline-.8 & 0 & -0.0014 & 0.0250 & 0.0500 & 0.0250 & -0.0150 & 0.0240 & 0.0435 & 0.0283 \\
\hline-.8 & .3 & 0.2991 & 0.0187 & 0.0505 & 0.0187 & 0.2878 & 0.0180 & 0.0430 & 0.0217 \\
\hline-.8 & .8 & 0.7997 & 0.0059 & 0.0480 & 0.0060 & 0.7973 & 0.0056 & 0.0310 & 0.0062 \\
\hline-.3 & -.8 & -0.8007 & 0.0214 & 0.0405 & 0.0214 & -0.7786 & 0.0179 & 0.0205 & 0.0279 \\
\hline-.3 & -.3 & -0.3014 & 0.0288 & 0.0475 & 0.0288 & -0.2992 & 0.0267 & 0.0110 & 0.0268 \\
\hline-.3 & 0 & -0.0011 & 0.0261 & 0.0470 & 0.0261 & -0.0062 & 0.0248 & 0.0200 & 0.0255 \\
\hline-.3 & .3 & 0.2995 & 0.0205 & 0.0480 & 0.0205 & 0.2920 & 0.0197 & 0.0295 & 0.0212 \\
\hline-.3 & .8 & 0.7997 & 0.0070 & 0.0465 & 0.0070 & 0.7974 & 0.0065 & 0.0310 & 0.0070 \\
\hline 0 & -.8 & -0.8002 & 0.0184 & 0.0390 & 0.0184 & -0.7786 & 0.0152 & 0.0235 & 0.0262 \\
\hline 0 & -.3 & -0.3004 & 0.0281 & 0.0420 & 0.0281 & -0.2924 & 0.0268 & 0.0085 & 0.0278 \\
\hline$\overline{0}$ & 0 & -0.0006 & 0.0273 & 0.0455 & 0.0273 & -0.0011 & 0.0263 & 0.0125 & 0.0263 \\
\hline 0 & .3 & 0.2995 & 0.0228 & 0.0440 & 0.0228 & 0.2950 & 0.0222 & 0.0245 & 0.0228 \\
\hline 0 & .8 & 0.7998 & 0.0083 & 0.0475 & 0.0083 & 0.7976 & 0.0078 & 0.0345 & 0.0082 \\
\hline .3 & -.8 & -0.7998 & 0.0163 & 0.0420 & 0.0163 & -0.7796 & 0.0136 & 0.0245 & 0.0245 \\
\hline .3 & -.3 & -0.2992 & 0.0291 & 0.0430 & 0.0291 & -0.2864 & 0.0279 & 0.0190 & 0.0311 \\
\hline .3 & 0 & 0.0003 & 0.0298 & 0.0435 & 0.0298 & 0.0046 & 0.0298 & 0.0175 & 0.0302 \\
\hline .3 & .3 & 0.3002 & 0.0263 & 0.0460 & 0.0263 & 0.2984 & 0.0262 & 0.0240 & 0.0263 \\
\hline .3 & .8 & 0.8004 & 0.0107 & 0.0500 & 0.0107 & 0.7978 & 0.0101 & 0.0410 & 0.0103 \\
\hline .8 & -.8 & -0.7994 & 0.0143 & 0.0525 & 0.0143 & -0.7830 & 0.0120 & 0.0170 & 0.0208 \\
\hline .8 & -.3 & -0.2967 & 0.0320 & 0.0600 & 0.0321 & -0.2747 & 0.0305 & 0.0260 & 0.0396 \\
\hline .8 & 0 & 0.0056 & 0.0385 & 0.0655 & 0.0389 & 0.0202 & 0.0384 & 0.0275 & 0.0434 \\
\hline .8 & .3 & 0.3075 & 0.0414 & 0.0725 & 0.0421 & 0.3118 & 0.0420 & 0.0350 & 0.0436 \\
\hline .8 & .8 & 0.8039 & 0.0268 & 0.0745 & 0.0271 & 0.7991 & 0.0279 & 0.0750 & 0.0279 \\
\hline & rage & 0.0004 & 0.0234 & 0.0496 & 0.0235 & 0.0041 & 0.0221 & 0.0273 & 0.0253 \\
\hline
\end{tabular}


Table 9: Heteroskedasticity with $\mathrm{c}=.5$, Modified Rook Matrix R2 (n=974)

\begin{tabular}{|c|c|c|c|c|c|c|c|c|c|}
\hline \multirow[b]{2}{*}{ rho } & \multirow[b]{2}{*}{ lambda } & \multicolumn{4}{|c|}{$\rho_{G S}$} & \multicolumn{4}{|c|}{$\rho_{M L}$} \\
\hline & & Median & Std. err & Rej. Rate & RMSE & Median & Std. err & Rej. Rate & RMSE \\
\hline-.8 & -.8 & -0.7976 & 0.0812 & 0.0460 & 0.0812 & -0.6273 & 0.0489 & 0.8935 & 0.1795 \\
\hline-.8 & -.3 & -0.7973 & 0.0784 & 0.0435 & 0.0785 & -0.6071 & 0.0486 & 0.9670 & 0.1989 \\
\hline-.8 & 0 & -0.7982 & 0.0771 & 0.0425 & 0.0771 & -0.6050 & 0.0486 & 0.9705 & 0.2009 \\
\hline-.8 & .3 & -0.7979 & 0.0770 & 0.0450 & 0.0770 & -0.6055 & 0.0484 & 0.9725 & 0.2005 \\
\hline-.8 & .8 & -0.7977 & 0.0760 & 0.0450 & 0.0760 & -0.6115 & 0.0481 & 0.9685 & 0.1945 \\
\hline-.3 & -.8 & -0.3006 & 0.0874 & 0.0495 & 0.0874 & -0.2652 & 0.0649 & 0.0530 & 0.0736 \\
\hline-.3 & -.3 & -0.3002 & 0.0871 & 0.0500 & 0.0871 & -0.2422 & 0.0641 & 0.0950 & 0.0863 \\
\hline-.3 & 0 & -0.3006 & 0.0870 & 0.0510 & 0.0870 & -0.2362 & 0.0644 & 0.1120 & 0.0906 \\
\hline-.3 & .3 & -0.3004 & 0.0870 & 0.0500 & 0.0870 & -0.2341 & 0.0648 & 0.1200 & 0.0924 \\
\hline-.3 & .8 & -0.2993 & 0.0844 & 0.0480 & 0.0844 & -0.2361 & 0.0631 & 0.1210 & 0.0898 \\
\hline 0 & -.8 & -0.0010 & 0.0826 & 0.0495 & 0.0826 & -0.0262 & 0.0683 & 0.0575 & 0.0731 \\
\hline 0 & -.3 & -0.0001 & 0.0842 & 0.0520 & 0.0842 & -0.0105 & 0.0674 & 0.0425 & 0.0682 \\
\hline 0 & 0 & 0.0003 & 0.0841 & 0.0520 & 0.0841 & -0.0038 & 0.0670 & 0.0435 & 0.0671 \\
\hline 0 & .3 & -0.0003 & 0.0843 & 0.0520 & 0.0843 & 0.0005 & 0.0677 & 0.0450 & 0.0677 \\
\hline 0 & .8 & -0.0014 & 0.0805 & 0.0510 & 0.0805 & 0.0007 & 0.0660 & 0.0495 & 0.0661 \\
\hline .3 & -.8 & 0.3004 & 0.0703 & 0.0550 & 0.0703 & 0.2378 & 0.0649 & 0.1735 & 0.0899 \\
\hline .3 & -.3 & 0.2988 & 0.0715 & 0.0500 & 0.0715 & 0.2449 & 0.0644 & 0.1475 & 0.0848 \\
\hline .3 & 0 & 0.2987 & 0.0729 & 0.0515 & 0.0729 & 0.2509 & 0.0645 & 0.1260 & 0.0811 \\
\hline .3 & .3 & 0.2985 & 0.0722 & 0.0530 & 0.0722 & 0.2559 & 0.0642 & 0.1090 & 0.0778 \\
\hline .3 & .8 & 0.2988 & 0.0711 & 0.0530 & 0.0711 & 0.2603 & 0.0623 & 0.1050 & 0.0739 \\
\hline .8 & -.8 & 0.7998 & 0.0342 & 0.0535 & 0.0342 & 0.7501 & 0.0387 & 0.3575 & 0.0632 \\
\hline .8 & -.3 & 0.7988 & 0.0361 & 0.0585 & 0.0361 & 0.7468 & 0.0400 & 0.3625 & 0.0666 \\
\hline .8 & 0 & 0.7977 & 0.0376 & 0.0570 & 0.0376 & 0.7473 & 0.0408 & 0.3345 & 0.0666 \\
\hline .8 & .3 & 0.7965 & 0.0393 & 0.0565 & 0.0395 & 0.7494 & 0.0432 & 0.2935 & 0.0665 \\
\hline .8 & .8 & 0.7942 & 0.0427 & 0.0545 & 0.0431 & 0.7546 & 0.0465 & 0.2460 & 0.0650 \\
\hline \multicolumn{2}{|c|}{ average } & -0.0004 & 0.0714 & 0.0508 & 0.0715 & 0.0275 & 0.0572 & 0.3106 & 0.0994 \\
\hline \multirow[b]{2}{*}{ rho } & \multirow[b]{2}{*}{ lambda } & & \multicolumn{4}{|c|}{$\lambda_{M L}$} \\
\hline & & \multicolumn{4}{|c|}{\begin{tabular}{c|c}
\multicolumn{2}{c}{$\lambda_{G S}$} \\
Std. err Rej. Rate
\end{tabular}} & Median & Std. err & Rej. Rate & RMSE \\
\hline-.8 & -.8 & -0.8005 & 0.0270 & 0.0455 & 0.0270 & -0.7845 & 0.0209 & 0.0450 & 0.0260 \\
\hline-.8 & -.3 & -0.2995 & 0.0259 & 0.0515 & 0.0259 & -0.3125 & 0.0227 & 0.0580 & 0.0259 \\
\hline-.8 & 0 & 0.0004 & 0.0219 & 0.0480 & 0.0219 & -0.0153 & 0.0203 & 0.0870 & 0.0254 \\
\hline-.8 & .3 & 0.3002 & 0.0163 & 0.0495 & 0.0163 & 0.2870 & 0.0157 & 0.0970 & 0.0204 \\
\hline-.8 & .8 & 0.8000 & 0.0056 & 0.0495 & 0.0056 & 0.7968 & 0.0053 & 0.0630 & 0.0062 \\
\hline-.3 & -.8 & -0.7996 & 0.0203 & 0.0495 & 0.0203 & -0.7720 & 0.0165 & 0.1370 & 0.0325 \\
\hline-.3 & -.3 & -0.2992 & 0.0243 & 0.0445 & 0.0243 & -0.2977 & 0.0224 & 0.0250 & 0.0225 \\
\hline-.3 & 0 & 0.0006 & 0.0219 & 0.0440 & 0.0219 & -0.0057 & 0.0208 & 0.0365 & 0.0215 \\
\hline-.3 & .3 & 0.3003 & 0.0178 & 0.0460 & 0.0178 & 0.2918 & 0.0171 & 0.0535 & 0.0189 \\
\hline-.3 & .8 & 0.8002 & 0.0068 & 0.0470 & 0.0068 & 0.7971 & 0.0065 & 0.0565 & 0.0071 \\
\hline 0 & -.8 & -0.7995 & 0.0176 & 0.0480 & 0.0176 & -0.7716 & 0.0144 & 0.1660 & 0.0319 \\
\hline 0 & -.3 & -0.2991 & 0.0248 & 0.0480 & 0.0248 & -0.2901 & 0.0234 & 0.0400 & 0.0254 \\
\hline$\overline{0}$ & 0 & 0.0008 & 0.0237 & 0.0500 & 0.0237 & 0.0005 & 0.0228 & 0.0290 & 0.0228 \\
\hline 0 & .3 & 0.3007 & 0.0197 & 0.0460 & 0.0197 & 0.2954 & 0.0194 & 0.0415 & 0.0199 \\
\hline 0 & .8 & 0.8004 & 0.0080 & 0.0480 & 0.0080 & 0.7974 & 0.0077 & 0.0590 & 0.0081 \\
\hline .3 & -.8 & -0.7990 & 0.0160 & 0.0535 & 0.0161 & -0.7727 & 0.0128 & 0.1805 & 0.0301 \\
\hline .3 & -.3 & -0.2986 & 0.0247 & 0.0515 & 0.0247 & -0.2825 & 0.0244 & 0.0635 & 0.0300 \\
\hline .3 & 0 & 0.0013 & 0.0259 & 0.0490 & 0.0259 & 0.0067 & 0.0253 & 0.0405 & 0.0262 \\
\hline .3 & .3 & 0.3010 & 0.0231 & 0.0500 & 0.0231 & 0.2996 & 0.0227 & 0.0395 & 0.0227 \\
\hline .3 & .8 & 0.8007 & 0.0104 & 0.0480 & 0.0104 & 0.7977 & 0.0098 & 0.0615 & 0.0101 \\
\hline .8 & -.8 & -0.7995 & 0.0140 & 0.0595 & 0.0140 & $\begin{array}{l}-0.7780 \\
\end{array}$ & 0.0101 & 0.1300 & 0.0243 \\
\hline .8 & -.3 & -0.2967 & 0.0265 & 0.0620 & 0.0267 & -0.2714 & 0.0242 & 0.0880 & 0.0375 \\
\hline .8 & 0 & 0.0049 & 0.0309 & 0.0665 & 0.0313 & 0.0228 & 0.0300 & 0.0705 & 0.0377 \\
\hline .8 & .3 & 0.3066 & 0.0333 & 0.0690 & 0.0340 & 0.3130 & 0.0329 & 0.0620 & 0.0354 \\
\hline .8 & .8 & 0.8038 & 0.0229 & 0.0690 & 0.0232 & 0.8005 & 0.0234 & 0.0895 & 0.0234 \\
\hline & rage & 0.0012 & 0.0204 & 0.0517 & 0.0204 & 0.0061 & 0.0189 & 0.0728 & 0.0237 \\
\hline
\end{tabular}


Table 10: Heteroskedasticity with $\mathrm{c}=.5$, Modified Rook Matrix R3 $(\mathrm{n}=485)$

\begin{tabular}{|c|c|c|c|c|c|c|c|c|c|}
\hline \multirow[b]{2}{*}{ rho } & \multirow[b]{2}{*}{ lambda } & \multicolumn{4}{|c|}{$\rho_{G S}$} & \multicolumn{4}{|c|}{$\rho_{M L}$} \\
\hline & & Median & Std. err & Rej. Rate & RMSE & Median & Std. err & Rej. Rate & RMSE \\
\hline-.8 & -.8 & -0.7910 & 0.0609 & 0.0555 & 0.0615 & -0.6728 & 0.0499 & 0.6015 & 0.1366 \\
\hline-.8 & -.3 & -0.7922 & 0.0557 & 0.0505 & 0.0562 & -0.6540 & 0.0489 & 0.8570 & 0.1539 \\
\hline-.8 & 0 & -0.7929 & 0.0540 & 0.0550 & 0.0544 & -0.6545 & 0.0483 & 0.8755 & 0.1534 \\
\hline-.8 & .3 & -0.7941 & 0.0527 & 0.0545 & 0.0530 & -0.6567 & 0.0473 & 0.8745 & 0.1509 \\
\hline-.8 & .8 & -0.7935 & 0.0517 & 0.0490 & 0.0521 & -0.6636 & 0.0457 & 0.8655 & 0.1439 \\
\hline-.3 & -.8 & -0.2877 & 0.0926 & 0.0620 & 0.0934 & -0.2575 & 0.0741 & 0.0830 & 0.0854 \\
\hline-.3 & -.3 & -0.2897 & 0.0935 & 0.0610 & 0.0941 & -0.2365 & 0.0708 & 0.1140 & 0.0950 \\
\hline-.3 & 0 & -0.2917 & 0.0932 & 0.0595 & 0.0936 & -0.2296 & 0.0706 & 0.1320 & 0.0997 \\
\hline-.3 & .3 & -0.2911 & 0.0924 & 0.0595 & 0.0928 & -0.2262 & 0.0708 & 0.1450 & 0.1023 \\
\hline-.3 & .8 & -0.2905 & 0.0903 & 0.0600 & 0.0908 & -0.2283 & 0.0704 & 0.1460 & 0.1004 \\
\hline 0 & -.8 & 0.0109 & 0.0975 & 0.0615 & 0.0981 & -0.0258 & 0.0853 & 0.0835 & 0.0892 \\
\hline 0 & -.3 & 0.0088 & 0.0989 & 0.0595 & 0.0993 & -0.0138 & 0.0810 & 0.0730 & 0.0822 \\
\hline 0 & 0 & 0.0065 & 0.0989 & 0.0605 & 0.0991 & -0.0060 & 0.0812 & 0.0735 & 0.0814 \\
\hline 0 & .3 & 0.0062 & 0.0996 & 0.0595 & 0.0998 & -0.0012 & 0.0814 & 0.0735 & 0.0814 \\
\hline 0 & .8 & 0.0076 & 0.0976 & 0.0590 & 0.0979 & -0.0014 & 0.0817 & 0.0775 & 0.0817 \\
\hline .3 & -.8 & 0.3083 & 0.0921 & 0.0630 & 0.0924 & 0.2259 & 0.0888 & 0.2275 & 0.1156 \\
\hline .3 & -.3 & 0.3047 & 0.0932 & 0.0610 & 0.0933 & 0.2288 & 0.0860 & 0.1990 & 0.1117 \\
\hline .3 & 0 & 0.3040 & 0.0926 & 0.0585 & 0.0927 & 0.2337 & 0.0842 & 0.1745 & 0.1072 \\
\hline .3 & .3 & 0.3025 & 0.0938 & 0.0585 & 0.0939 & 0.2391 & 0.0840 & 0.1605 & 0.1037 \\
\hline .3 & .8 & 0.3043 & 0.0937 & 0.0595 & 0.0938 & 0.2425 & 0.0850 & 0.1620 & 0.1026 \\
\hline .8 & -.8 & 0.8007 & 0.0536 & 0.0540 & 0.0536 & 0.7351 & 0.0574 & 0.4255 & 0.0867 \\
\hline .8 & -.3 & 0.7991 & 0.0538 & 0.0570 & 0.0538 & 0.7300 & 0.0588 & 0.4355 & 0.0914 \\
\hline .8 & 0 & 0.7973 & 0.0550 & 0.0580 & 0.0550 & 0.7293 & 0.0601 & 0.4220 & 0.0928 \\
\hline .8 & .3 & 0.7940 & 0.0565 & 0.0660 & 0.0568 & 0.7298 & 0.0606 & 0.3930 & 0.0927 \\
\hline .8 & .8 & 0.7919 & 0.0595 & 0.0600 & 0.0601 & 0.7321 & 0.0654 & 0.3475 & 0.0943 \\
\hline \multicolumn{2}{|c|}{ average } & 0.0053 & 0.0789 & 0.0585 & 0.0793 & 0.0119 & 0.0695 & 0.3209 & 0.1054 \\
\hline \multirow[b]{2}{*}{ rho } & \multirow[b]{2}{*}{ lambda } & \multicolumn{4}{|c|}{$\lambda_{G S}$} & \multicolumn{4}{|c|}{$\lambda_{M L}$} \\
\hline & & Median & Std. err & Rej. Rate & RMSE & \multicolumn{4}{|c|}{\begin{tabular}{l|l}
\multicolumn{3}{c}{$\lambda_{M L}$} \\
Std. err & Rej. Rate
\end{tabular}} \\
\hline-.8 & -.8 & -0.8037 & 0.0307 & 0.0640 & 0.0309 & -0.7916 & 0.0244 & 0.0230 & 0.0258 \\
\hline-.8 & -.3 & -0.3020 & 0.0355 & 0.0555 & 0.0355 & -0.3189 & 0.0315 & 0.0840 & 0.0367 \\
\hline-.8 & 0 & -0.0008 & 0.0303 & 0.0520 & 0.0303 & -0.0227 & 0.0287 & 0.1240 & 0.0366 \\
\hline-.8 & .3 & 0.2997 & 0.0230 & 0.0525 & 0.0230 & 0.2811 & 0.0223 & 0.1410 & 0.0292 \\
\hline-.8 & .8 & 0.8000 & 0.0077 & 0.0570 & 0.0077 & 0.7959 & 0.0070 & 0.0890 & 0.0081 \\
\hline-.3 & -.8 & -0.8005 & 0.0156 & 0.0455 & 0.0156 & -0.7808 & 0.0146 & 0.0635 & 0.0241 \\
\hline-.3 & -.3 & -0.3006 & 0.0269 & 0.0480 & 0.0269 & -0.2981 & 0.0242 & 0.0180 & 0.0242 \\
\hline-.3 & 0 & -0.0001 & 0.0270 & 0.0495 & 0.0270 & -0.0078 & 0.0251 & 0.0390 & 0.0262 \\
\hline-.3 & .3 & 0.3001 & 0.0227 & 0.0535 & 0.0227 & 0.2884 & 0.0217 & 0.0670 & 0.0246 \\
\hline-.3 & .8 & 0.8002 & 0.0084 & 0.0550 & 0.0084 & 0.7960 & 0.0079 & 0.0825 & 0.0089 \\
\hline 0 & -.8 & -0.8001 & 0.0127 & 0.0445 & 0.0127 & -0.7817 & 0.0120 & 0.0910 & 0.0219 \\
\hline 0 & -.3 & -0.2999 & 0.0241 & 0.0510 & 0.0241 & -0.2898 & 0.0226 & 0.0220 & 0.0248 \\
\hline 0 & 0 & 0.0001 & 0.0255 & 0.0500 & 0.0255 & -0.0004 & 0.0247 & 0.0255 & 0.0247 \\
\hline 0 & .3 & 0.3001 & 0.0228 & 0.0500 & 0.0228 & 0.2933 & 0.0225 & 0.0450 & 0.0234 \\
\hline 0 & .8 & 0.8004 & 0.0095 & 0.0575 & 0.0095 & 0.7961 & 0.0091 & 0.0750 & 0.0099 \\
\hline .3 & -.8 & -0.8000 & 0.0114 & 0.0495 & 0.0114 & -0.7833 & 0.0107 & 0.1090 & 0.0198 \\
\hline .3 & -.3 & -0.2992 & 0.0237 & 0.0495 & 0.0237 & -0.2834 & 0.0231 & 0.0460 & 0.0285 \\
\hline .3 & 0 & 0.0012 & 0.0271 & 0.0530 & 0.0272 & 0.0078 & 0.0263 & 0.0360 & 0.0275 \\
\hline .3 & .3 & 0.3010 & 0.0252 & 0.0550 & 0.0253 & 0.2988 & 0.0248 & 0.0435 & 0.0248 \\
\hline .3 & .8 & 0.8005 & 0.0119 & 0.0530 & 0.0119 & 0.7965 & 0.0117 & 0.0770 & 0.0122 \\
\hline .8 & -.8 & -0.7998 & 0.0115 & 0.0515 & 0.0115 & -0.7863 & 0.0101 & 0.1165 & 0.0170 \\
\hline .8 & -.3 & -0.2976 & 0.0269 & 0.0565 & 0.0270 & -0.2741 & 0.0256 & 0.0945 & 0.0365 \\
\hline .8 & 0 & 0.0044 & 0.0340 & 0.0615 & 0.0343 & 0.0220 & 0.0327 & 0.0675 & 0.0394 \\
\hline .8 & .3 & 0.3066 & 0.0384 & 0.0740 & 0.0390 & 0.3134 & 0.0369 & 0.0565 & 0.0392 \\
\hline .8 & .8 & 0.8047 & 0.0269 & 0.0740 & 0.0273 & 0.7997 & 0.0282 & 0.1240 & 0.0282 \\
\hline \multicolumn{2}{|c|}{ average } & 0.0006 & 0.0224 & 0.0545 & 0.0224 & 0.0028 & 0.0211 & 0.0704 & 0.0249 \\
\hline
\end{tabular}


Table 11: Heteroskedasticity with $\mathrm{c}=.5$, Modified Rook Matrix R4 $(\mathrm{n}=945)$

\begin{tabular}{|c|c|c|c|c|c|c|c|c|c|}
\hline \multirow[b]{2}{*}{ rho } & \multirow[b]{2}{*}{ lambda } & \multicolumn{4}{|c|}{$\rho_{G S}$} & \multicolumn{4}{|c|}{$\rho_{M L}$} \\
\hline & & Median & Std. err & Rej. Rate & RMSE & Median & Std. err & Rej. Rate & RMSE \\
\hline-.8 & -.8 & -0.7967 & 0.0454 & 0.0440 & 0.0455 & -0.6720 & 0.0372 & 0.9220 & 0.1333 \\
\hline-.8 & -.3 & -0.7981 & 0.0408 & 0.0440 & 0.0409 & -0.6503 & 0.0345 & 0.9965 & 0.1536 \\
\hline-.8 & 0 & -0.7987 & 0.0388 & 0.0475 & 0.0388 & -0.6496 & 0.0339 & 0.9975 & 0.1542 \\
\hline-.8 & .3 & -0.7991 & 0.0384 & 0.0475 & 0.0385 & -0.6517 & 0.0338 & 0.9970 & 0.1521 \\
\hline-.8 & .8 & -0.7991 & 0.0378 & 0.0465 & 0.0378 & -0.6590 & 0.0330 & 0.9960 & 0.1448 \\
\hline-.3 & -.8 & -0.2954 & 0.0698 & 0.0470 & 0.0699 & -0.2540 & 0.0548 & 0.1145 & 0.0715 \\
\hline-.3 & -.3 & -0.2967 & 0.0701 & 0.0525 & 0.0702 & -0.2308 & 0.0517 & 0.2115 & 0.0864 \\
\hline-.3 & 0 & -0.2961 & 0.0686 & 0.0520 & 0.0687 & -0.2216 & 0.0513 & 0.2610 & 0.0937 \\
\hline-.3 & .3 & -0.2965 & 0.0673 & 0.0520 & 0.0674 & -0.2174 & 0.0515 & 0.2975 & 0.0973 \\
\hline-.3 & .8 & -0.2965 & 0.0661 & 0.0485 & 0.0662 & -0.2221 & 0.0523 & 0.2870 & 0.0939 \\
\hline 0 & -.8 & 0.0067 & 0.0739 & 0.0465 & 0.0742 & -0.0233 & 0.0618 & 0.0865 & 0.0661 \\
\hline 0 & -.3 & 0.0040 & 0.0746 & 0.0510 & 0.0747 & -0.0099 & 0.0595 & 0.0595 & 0.0603 \\
\hline 0 & 0 & 0.0032 & 0.0737 & 0.0550 & 0.0737 & -0.0013 & 0.0590 & 0.0610 & 0.0590 \\
\hline 0 & .3 & 0.0039 & 0.0735 & 0.0535 & 0.0736 & 0.0053 & 0.0596 & 0.0595 & 0.0598 \\
\hline 0 & .8 & 0.0043 & 0.0724 & 0.0485 & 0.0725 & 0.0064 & 0.0604 & 0.0650 & 0.0607 \\
\hline .3 & -.8 & 0.3067 & 0.0699 & 0.0480 & 0.0702 & 0.2271 & 0.0640 & 0.3240 & 0.0970 \\
\hline .3 & -.3 & 0.3055 & 0.0703 & 0.0485 & 0.0705 & 0.2301 & 0.0628 & 0.2905 & 0.0939 \\
\hline .3 & 0 & 0.3051 & 0.0703 & 0.0485 & 0.0705 & 0.2376 & 0.0620 & 0.2545 & 0.0880 \\
\hline .3 & .3 & 0.3042 & 0.0706 & 0.0510 & 0.0707 & 0.2453 & 0.0619 & 0.2110 & 0.0826 \\
\hline .3 & .8 & 0.3035 & 0.0710 & 0.0495 & 0.0711 & 0.2510 & 0.0627 & 0.2000 & 0.0796 \\
\hline .8 & -.8 & 0.8020 & 0.0392 & 0.0430 & 0.0392 & 0.7342 & 0.0434 & 0.6165 & 0.0789 \\
\hline .8 & -.3 & 0.8017 & 0.0393 & 0.0445 & 0.0394 & 0.7294 & 0.0436 & 0.6380 & 0.0830 \\
\hline .8 & 0 & 0.8008 & 0.0395 & 0.0465 & 0.0395 & 0.7295 & 0.0445 & 0.6150 & 0.0834 \\
\hline .8 & .3 & 0.7991 & 0.0402 & 0.0430 & 0.0402 & 0.7316 & 0.0446 & 0.5640 & 0.0816 \\
\hline .8 & .8 & 0.7987 & 0.0432 & 0.0450 & 0.0433 & 0.7376 & 0.0484 & 0.4360 & 0.0789 \\
\hline \multicolumn{2}{|c|}{ average } & 0.0031 & 0.0586 & 0.0481 & 0.0587 & 0.0161 & 0.0509 & 0.4225 & 0.0933 \\
\hline \multirow[b]{2}{*}{ rho } & \multirow[b]{2}{*}{ lambda } & & \multicolumn{4}{|c|}{$\lambda_{M L}$} \\
\hline & & \multicolumn{4}{|c|}{\begin{tabular}{c|c}
\multicolumn{2}{c}{$\lambda_{G S}$} \\
Std. err Rej. Rate
\end{tabular}} & Median & Std. err & Rej. Rate & RMSE \\
\hline-.8 & -.8 & -0.8028 & 0.0233 & 0.0565 & 0.0235 & -0.7906 & 0.0186 & 0.0360 & 0.0208 \\
\hline-.8 & -.3 & -0.3012 & 0.0279 & 0.0515 & 0.0280 & -0.3199 & 0.0241 & 0.1175 & 0.0313 \\
\hline-.8 & 0 & -0.0004 & 0.0245 & 0.0500 & 0.0245 & -0.0250 & 0.0222 & 0.2025 & 0.0334 \\
\hline-.8 & .3 & 0.3000 & 0.0186 & 0.0510 & 0.0186 & 0.2788 & 0.0177 & 0.2295 & 0.0276 \\
\hline-.8 & .8 & 0.8002 & 0.0065 & 0.0515 & 0.0065 & 0.7949 & 0.0059 & 0.1405 & 0.0078 \\
\hline-.3 & -.8 & -0.8007 & 0.0110 & 0.0525 & 0.0111 & -0.7793 & 0.0104 & 0.1985 & 0.0232 \\
\hline-.3 & -.3 & -0.3006 & 0.0201 & 0.0490 & 0.0201 & -0.2968 & 0.0179 & 0.0195 & 0.0182 \\
\hline-.3 & 0 & -0.0006 & 0.0205 & 0.0465 & 0.0205 & -0.0090 & 0.0190 & 0.0440 & 0.0211 \\
\hline-.3 & .3 & 0.2997 & 0.0177 & 0.0515 & 0.0177 & 0.2860 & 0.0163 & 0.1045 & 0.0215 \\
\hline-.3 & .8 & 0.8001 & 0.0071 & 0.0505 & 0.0071 & 0.7947 & 0.0067 & 0.1195 & 0.0085 \\
\hline 0 & -.8 & -0.8004 & 0.0093 & 0.0515 & 0.0093 & -0.7805 & 0.0086 & 0.2580 & 0.0213 \\
\hline 0 & -.3 & -0.3006 & 0.0180 & 0.0515 & 0.0181 & -0.2883 & 0.0168 & 0.0390 & 0.0204 \\
\hline$\overline{0}$ & 0 & -0.0003 & 0.0198 & 0.0500 & 0.0198 & -0.0005 & 0.0187 & 0.0230 & 0.0187 \\
\hline 0 & .3 & 0.2997 & 0.0176 & 0.0450 & 0.0176 & 0.2914 & 0.0168 & 0.0480 & 0.0189 \\
\hline 0 & .8 & 0.8001 & 0.0081 & 0.0495 & 0.0081 & 0.7949 & 0.0076 & 0.0955 & 0.0091 \\
\hline .3 & -.8 & -0.8003 & 0.0086 & 0.0510 & 0.0086 & -0.7826 & 0.0077 & 0.2845 & 0.0190 \\
\hline .3 & -.3 & -0.3008 & 0.0180 & 0.0545 & 0.0180 & -0.2814 & 0.0171 & 0.0740 & 0.0253 \\
\hline .3 & 0 & -0.0005 & 0.0200 & 0.0525 & 0.0200 & 0.0082 & 0.0193 & 0.0370 & 0.0210 \\
\hline .3 & .3 & 0.2997 & 0.0197 & 0.0485 & 0.0197 & 0.2975 & 0.0192 & 0.0275 & 0.0194 \\
\hline .3 & .8 & 0.8002 & 0.0097 & 0.0480 & 0.0097 & 0.7952 & 0.0095 & 0.0770 & 0.0107 \\
\hline .8 & -.8 & -0.8004 & 0.0080 & 0.0550 & 0.0081 & -0.7869 & 0.0071 & 0.2560 & 0.0150 \\
\hline .8 & -.3 & -0.3002 & 0.0193 & 0.0505 & 0.0193 & -0.2732 & 0.0185 & 0.1690 & 0.0326 \\
\hline .8 & 0 & 0.0011 & 0.0242 & 0.0515 & 0.0243 & 0.0231 & 0.0233 & 0.0905 & 0.0329 \\
\hline .8 & .3 & 0.3023 & 0.0271 & 0.0565 & 0.0272 & 0.3133 & 0.0264 & 0.0395 & 0.0296 \\
\hline .8 & .8 & 0.8017 & 0.0201 & 0.0545 & 0.0202 & 0.7985 & 0.0214 & 0.0690 & 0.0214 \\
\hline & rage & -0.0002 & 0.0170 & 0.0512 & 0.0170 & 0.0025 & 0.0159 & 0.1120 & 0.0211 \\
\hline
\end{tabular}


Table 12: Heteroskedasticity with $\mathrm{c}=.5$, Circular Matrix $(\mathrm{n}=500)$

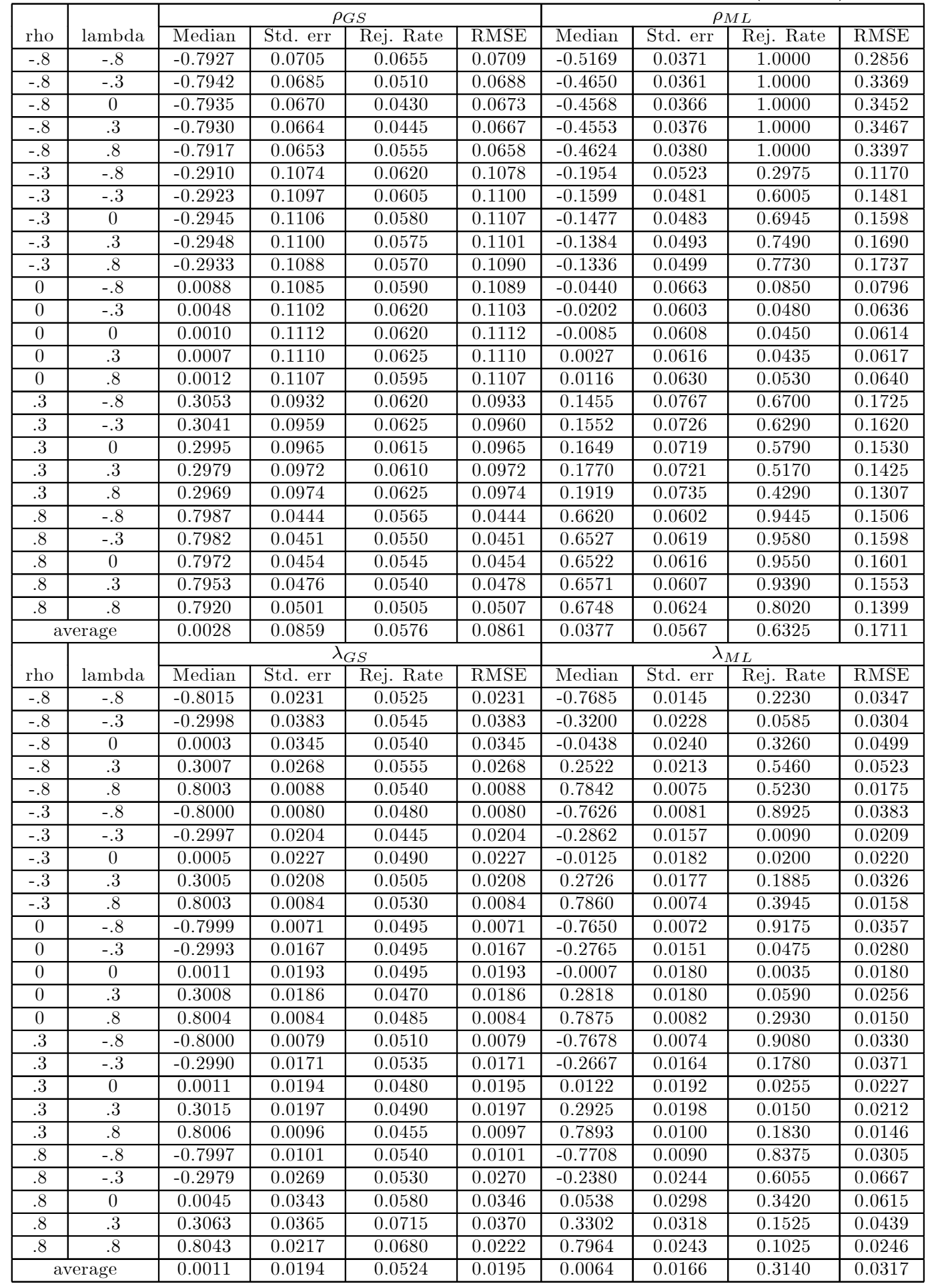


Table 13: Heteroskedasticity with $\mathrm{c}=.5$, Circular Matrix ( $\mathrm{n}=1000)$

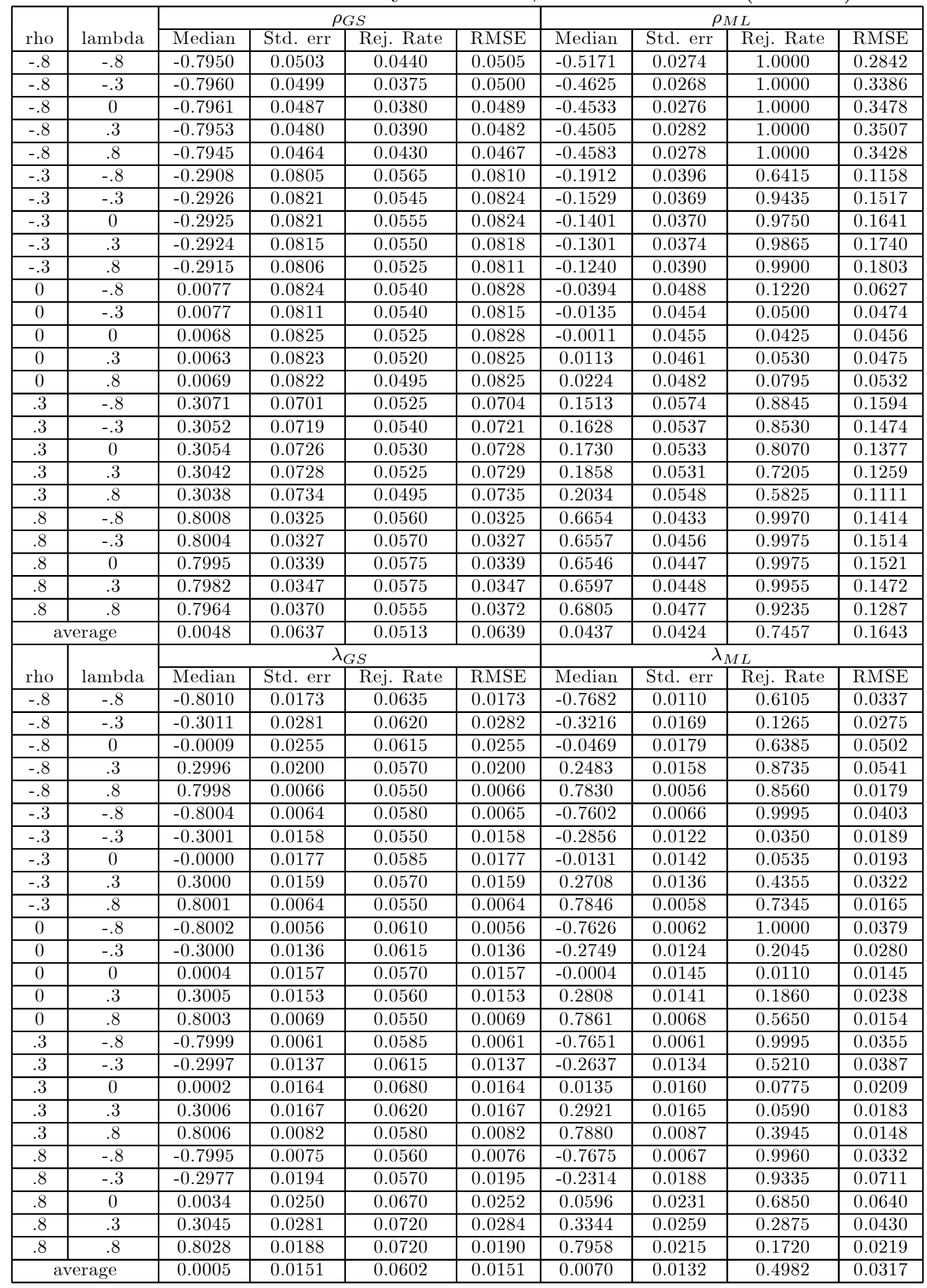


Table 14: Homoskedasticity with $\mathrm{c}=1$, Modified Rook Matrix R1 ( $\mathrm{n}=486$ )

\begin{tabular}{|c|c|c|c|c|c|c|c|c|c|}
\hline \multirow[b]{2}{*}{ rho } & \multirow[b]{2}{*}{ lambda } & \multicolumn{4}{|c|}{$\rho_{G S}$} & \multicolumn{4}{|c|}{$\rho_{M L}$} \\
\hline & & Median & Std. err & Rej. Rate & RMSE & Median & Std. err & Rej. Rate & RMSE \\
\hline-.8 & -.8 & -0.7724 & 0.1019 & 0.0895 & 0.1056 & -0.7932 & 0.0855 & 0.0815 & 0.0858 \\
\hline-.8 & -.3 & -0.7939 & 0.0704 & 0.0555 & 0.0707 & -0.7970 & 0.0588 & 0.0455 & 0.0589 \\
\hline-.8 & 0 & -0.7979 & 0.0642 & 0.0485 & 0.0643 & -0.7966 & 0.0565 & 0.0545 & 0.0566 \\
\hline-.8 & .3 & -0.7994 & 0.0610 & 0.0480 & 0.0610 & -0.7970 & 0.0532 & 0.0580 & 0.0533 \\
\hline-.8 & .8 & -0.8003 & 0.0576 & 0.0550 & 0.0576 & -0.7978 & 0.0513 & 0.0590 & 0.0514 \\
\hline-.3 & -.8 & -0.2926 & 0.1124 & 0.0555 & 0.1127 & -0.3038 & 0.1108 & 0.0450 & 0.1108 \\
\hline-.3 & -.3 & -0.2981 & 0.1052 & 0.0500 & 0.1052 & -0.3022 & 0.1031 & 0.0475 & 0.1031 \\
\hline-.3 & 0 & -0.3010 & 0.1032 & 0.0505 & 0.1032 & -0.3026 & 0.0989 & 0.0475 & 0.0990 \\
\hline-.3 & .3 & -0.3026 & 0.0975 & 0.0510 & 0.0975 & -0.3026 & 0.0956 & 0.0520 & 0.0956 \\
\hline-.3 & .8 & -0.3033 & 0.0901 & 0.0545 & 0.0901 & -0.3019 & 0.0890 & 0.0545 & 0.0890 \\
\hline 0 & -.8 & 0.0015 & 0.1038 & 0.0490 & 0.1038 & -0.0048 & 0.1023 & 0.0420 & 0.1024 \\
\hline 0 & -.3 & -0.0015 & 0.1069 & 0.0510 & 0.1069 & -0.0041 & 0.1048 & 0.0465 & 0.1049 \\
\hline 0 & 0 & -0.0038 & 0.1054 & 0.0490 & 0.1055 & -0.0037 & 0.1029 & 0.0485 & 0.1030 \\
\hline 0 & .3 & -0.0059 & 0.1036 & 0.0500 & 0.1037 & -0.0041 & 0.1019 & 0.0480 & 0.1020 \\
\hline 0 & .8 & -0.0063 & 0.0935 & 0.0535 & 0.0937 & -0.0035 & 0.0924 & 0.0515 & 0.0925 \\
\hline .3 & -.8 & 0.2994 & 0.0885 & 0.0495 & 0.0885 & 0.2975 & 0.0871 & 0.0455 & 0.0872 \\
\hline .3 & -.3 & 0.2960 & 0.0944 & 0.0545 & 0.0945 & 0.2980 & 0.0940 & 0.0530 & 0.0940 \\
\hline .3 & 0 & 0.2938 & 0.0949 & 0.0550 & 0.0951 & 0.2958 & 0.0954 & 0.0465 & 0.0955 \\
\hline .3 & .3 & 0.2924 & 0.0959 & 0.0495 & 0.0962 & 0.2950 & 0.0948 & 0.0460 & 0.0949 \\
\hline .3 & .8 & 0.2933 & 0.0884 & 0.0485 & 0.0887 & 0.2967 & 0.0878 & 0.0470 & 0.0879 \\
\hline .8 & -.8 & 0.7995 & 0.0427 & 0.0500 & 0.0427 & 0.7972 & 0.0399 & 0.0530 & 0.0400 \\
\hline .8 & -.3 & 0.7956 & 0.0503 & 0.0580 & 0.0505 & 0.7960 & 0.0448 & 0.0500 & 0.0449 \\
\hline .8 & 0 & 0.7906 & 0.0576 & 0.0635 & 0.0583 & 0.7955 & 0.0503 & 0.0525 & 0.0505 \\
\hline .8 & .3 & 0.7841 & 0.0657 & 0.0720 & 0.0676 & 0.7939 & 0.0564 & 0.0560 & 0.0568 \\
\hline .8 & .8 & 0.7791 & 0.0656 & 0.0660 & 0.0688 & 0.7962 & 0.0674 & 0.0900 & 0.0675 \\
\hline \multicolumn{2}{|c|}{ average } & -0.0021 & 0.0848 & 0.0551 & 0.0853 & -0.0021 & 0.0810 & 0.0528 & 0.0811 \\
\hline \multirow[b]{2}{*}{ rho } & \multirow[b]{2}{*}{ lambda } & \multicolumn{4}{|c|}{$\lambda_{G S}$} & \multicolumn{4}{|c|}{$\lambda_{M L}$} \\
\hline & & \multicolumn{4}{|c|}{\begin{tabular}{c|c}
\multicolumn{2}{c}{$\lambda_{G S}$} \\
Std. err Rej. Rate
\end{tabular}} & \multicolumn{4}{|c|}{\begin{tabular}{l}
\multicolumn{2}{c}{$\lambda_{M L}$} \\
Std. err $\mid$ Rej. Rate
\end{tabular}} \\
\hline-.8 & -.8 & -0.8282 & 0.0736 & 0.1220 & 0.0788 & -0.8010 & 0.0689 & 0.0835 & 0.0689 \\
\hline-.8 & -.3 & -0.3105 & 0.0640 & 0.0655 & 0.0649 & -0.3053 & 0.0584 & 0.0480 & 0.0587 \\
\hline-.8 & 0 & -0.0041 & 0.0510 & 0.0550 & 0.0511 & -0.0041 & 0.0484 & 0.0425 & 0.0486 \\
\hline-.8 & .3 & 0.2990 & 0.0373 & 0.0520 & 0.0373 & 0.2973 & 0.0358 & 0.0455 & 0.0359 \\
\hline-.8 & .8 & 0.8001 & 0.0119 & 0.0515 & 0.0119 & 0.7990 & 0.0112 & 0.0425 & 0.0112 \\
\hline-.3 & -.8 & -0.8081 & 0.0542 & 0.0600 & 0.0548 & -0.7975 & 0.0497 & 0.0520 & 0.0498 \\
\hline-.3 & -.3 & -0.3051 & 0.0619 & 0.0550 & 0.0621 & -0.3017 & 0.0618 & 0.0500 & 0.0618 \\
\hline-.3 & 0 & -0.0021 & 0.0537 & 0.0505 & 0.0537 & -0.0026 & 0.0528 & 0.0465 & 0.0528 \\
\hline-.3 & .3 & 0.2996 & 0.0417 & 0.0495 & 0.0417 & 0.2981 & 0.0400 & 0.0440 & 0.0400 \\
\hline-.3 & .8 & 0.8000 & 0.0140 & 0.0495 & 0.0140 & 0.7990 & 0.0132 & 0.0445 & 0.0133 \\
\hline 0 & -.8 & -0.8040 & 0.0479 & 0.0545 & 0.0481 & -0.7971 & 0.0424 & 0.0450 & 0.0425 \\
\hline 0 & -.3 & -0.3019 & 0.0638 & 0.0535 & 0.0639 & -0.3011 & 0.0639 & 0.0480 & 0.0639 \\
\hline 0 & 0 & -0.0017 & 0.0587 & 0.0525 & 0.0587 & -0.0018 & 0.0578 & 0.0495 & 0.0578 \\
\hline 0 & .3 & 0.2997 & 0.0464 & 0.0510 & 0.0464 & 0.2980 & 0.0459 & 0.0460 & 0.0460 \\
\hline 0 & .8 & 0.8002 & 0.0165 & 0.0480 & 0.0165 & 0.7984 & 0.0154 & 0.0430 & 0.0155 \\
\hline .3 & -.8 & -0.8019 & 0.0448 & 0.0515 & 0.0449 & -0.7977 & 0.0387 & 0.0455 & 0.0388 \\
\hline .3 & -.3 & -0.2987 & 0.0676 & 0.0600 & 0.0676 & -0.2988 & 0.0683 & 0.0475 & 0.0683 \\
\hline .3 & 0 & 0.0018 & 0.0660 & 0.0575 & 0.0660 & 0.0003 & 0.0666 & 0.0500 & 0.0666 \\
\hline .3 & .3 & 0.3020 & 0.0553 & 0.0595 & 0.0554 & 0.2983 & 0.0551 & 0.0510 & 0.0551 \\
\hline .3 & .8 & 0.8006 & 0.0211 & 0.0485 & 0.0212 & 0.7980 & 0.0202 & 0.0460 & 0.0203 \\
\hline .8 & -.8 & -0.7994 & 0.0403 & 0.0555 & 0.0403 & -0.7965 & 0.0321 & 0.0515 & 0.0323 \\
\hline .8 & -.3 & -0.2894 & 0.0820 & 0.0765 & 0.0827 & -0.2946 & 0.0698 & 0.0550 & 0.0700 \\
\hline .8 & 0 & 0.0188 & 0.0974 & 0.0940 & 0.0992 & 0.0055 & 0.0808 & 0.0575 & 0.0810 \\
\hline .8 & .3 & 0.3276 & 0.0997 & 0.1055 & 0.1034 & 0.3039 & 0.0867 & 0.0695 & 0.0868 \\
\hline .8 & .8 & 0.8176 & 0.0486 & 0.1045 & 0.0517 & 0.7991 & 0.0525 & 0.0915 & 0.0525 \\
\hline \multicolumn{2}{|c|}{ average } & 0.0005 & 0.0528 & 0.0633 & 0.0534 & -0.0002 & 0.0495 & 0.0518 & 0.0495 \\
\hline
\end{tabular}




\section{A Appendix: Additional Assumptions}

In this appendix we state the additional assumptions needed to formally establish the limiting distribution of the GMM/IV estimators.

Assumption A.1 Let $\Gamma_{n}=\left[\gamma_{r s, n}\right]_{r, s=1,2}$ and $\gamma_{n}=\left[\gamma_{1, n}, \gamma_{2, n}\right]^{\prime}$ where, dropping the subscript $n$ temporarily for notational convenience,

$$
\begin{aligned}
& \gamma_{11}=2 n^{-1} E\left\{\overline{\bar{u}}^{\prime} \bar{u}-\operatorname{Tr}\left[M\left[\operatorname{diag}_{i=1}^{n}\left(\bar{u}_{i} u_{i}\right)\right] M^{\prime}\right]\right\}=2 n^{-1} E u^{\prime} M^{\prime} A_{1} u \\
& \gamma_{12}=-n^{-1} E\left\{\overline{\bar{u}}^{\prime} \overline{\bar{u}}+\operatorname{Tr}\left[M\left[\operatorname{diag}_{i=1}^{n}\left(\bar{u}_{i}^{2}\right)\right] M^{\prime}\right]\right\}=-n^{-1} E u^{\prime} M^{\prime} A_{1} M u, \\
& \gamma_{21}=n^{-1} E\left(u^{\prime} \overline{\bar{u}}+\bar{u}^{\prime} \bar{u}\right)=n^{-1} E u^{\prime} M^{\prime}\left(A_{2}+A_{2}^{\prime}\right) u \\
& \gamma_{22}=-n^{-1} E \bar{u}^{\prime} \overline{\bar{u}}=-n^{-1} E u^{\prime} M^{\prime} A_{2} M u, \\
& \gamma_{1}=n^{-1} E\left\{\bar{u}^{\prime} \bar{u}-\operatorname{Tr}\left[M\left[\operatorname{diag}_{i=1}^{n}\left(u_{i}^{2}\right)\right] M^{\prime}\right]\right\}=n^{-1} E u^{\prime} A_{1} u, \\
& \gamma_{2}=n^{-1} E u^{\prime} \bar{u}=n^{-1} E u^{\prime} A_{2} u
\end{aligned}
$$

with $\bar{u}=M u$, and $\overline{\bar{u}}=M \bar{u}=M^{2} u$. Then $\Gamma_{n}$ is nonsingular for all $n$ sufficiently large and $\lim _{n \rightarrow \infty} \Gamma_{n}=\Gamma$ is finite and nonsingular.

Assumption A.2 Let $\Psi_{n}=\left(\psi_{r s, n}\right)$ where for $r, s=1,2$

$$
\psi_{r s, n}=(2 n)^{-1} \operatorname{tr}\left[\left(A_{r, n}+A_{r, n}^{\prime}\right) \boldsymbol{\Sigma}_{n}\left(A_{s, n}+A_{s, n}^{\prime}\right) \Sigma_{n}\right]+n^{-1} a_{r, n}^{\prime} \boldsymbol{\Sigma}_{n} a_{s, n}
$$

with $a_{r, n}=\left(I_{n}-\rho M_{n}^{\prime}\right)^{-1} H_{n} P \alpha_{r, n}$ where

$$
\begin{aligned}
\alpha_{r, n} & =-n^{-1} E\left[Z_{n}^{\prime}\left(I_{n}-\rho M_{n}^{\prime}\right)\left(A_{r, n}+A_{r, n}^{\prime}\right)\left(I_{n}-\rho M_{n}\right) u_{n}\right], \\
P & =Q_{H H}^{-1} Q_{H Z}\left[Q_{H Z}^{\prime} Q_{H H}^{-1} Q_{H Z}\right]^{-1},
\end{aligned}
$$

and $\boldsymbol{\Sigma}_{n}=\operatorname{diag}\left(\sigma_{i, n}^{2}\right)$, where $\sigma_{i, n}^{2}=E \varepsilon_{i, n}^{2}$. Furthermore, let

$$
\Psi_{\circ, n}=\left[\begin{array}{cc}
\Psi_{\Delta \Delta, n} & \Psi_{\Delta \rho, n} \\
\Psi_{\Delta \rho, n}^{\prime} & \Psi_{n}
\end{array}\right]
$$

with

$$
\begin{aligned}
\Psi_{\Delta \Delta, n} & =n^{-1} H_{n}^{\prime}\left(I_{n}-\rho M_{n}\right)^{-1} \Sigma_{n}\left(I_{n}-\rho M_{n}^{\prime}\right)^{-1} H_{n}, \\
\Psi_{\Delta \rho, n} & =n^{-1} H_{n}^{\prime}\left(I_{n}-\rho M_{n}\right)^{-1} \Sigma_{n}\left[a_{1, n}, a_{2, n}\right] .
\end{aligned}
$$

Then $\Psi_{n}$ and $\Psi_{\circ, n}$ are nonsingular for all $n$ sufficiently large and $\lim _{n \rightarrow \infty} \Psi_{n}=$ $\Psi$ and $\lim _{n \rightarrow \infty} \Psi_{\circ, n}=\Psi_{\circ}$ are finite and nonsingular.

Assumption A.3 Let $\Psi_{n}=\left(\psi_{r s, n}\right)$ where for $r, s=1,2$

$$
\psi_{r s, n}=(2 n)^{-1} \operatorname{tr}\left[\left(A_{r, n}+A_{r, n}^{\prime}\right) \boldsymbol{\Sigma}_{n}\left(A_{s, n}+A_{s, n}^{\prime}\right) \Sigma_{n}\right]+n^{-1} a_{r, n}^{\prime} \boldsymbol{\Sigma}_{n} a_{s, n}
$$

with

$$
\begin{aligned}
a_{r, n} & =H_{n} P \alpha_{r, n}, \\
\alpha_{r, n} & =-n^{-1} E\left[Z_{n}^{\prime}\left(I_{n}-\rho M_{n}^{\prime}\right)\left(A_{r, n}+A_{r, n}^{\prime}\right)\left(I_{n}-\rho M_{n}\right) u_{n}\right], \\
P & =Q_{H H}^{-1} Q_{H Z *}\left[Q_{H Z *}^{\prime} Q_{H H}^{-1} Q_{H Z *}\right]^{-1},
\end{aligned}
$$


and $\boldsymbol{\Sigma}_{n}=\operatorname{diag}\left(\sigma_{i, n}^{2}\right)$, where $\sigma_{i, n}^{2}=E \varepsilon_{i, n}^{2}$. Furthermore, let

$$
\Psi_{\circ, n}=\left[\begin{array}{cc}
\Psi_{\delta \delta, n} & \Psi_{\delta \rho, n} \\
\Psi_{\delta \rho, n}^{\prime} & \Psi_{n}
\end{array}\right]
$$

with

$$
\begin{aligned}
\Psi_{\delta \delta, n} & =n^{-1} H_{n}^{\prime} \Sigma_{n} H_{n}, \\
\Psi_{\delta \rho, n} & =n^{-1} H_{n}^{\prime} \Sigma_{n}\left[a_{1, n}, a_{2, n}\right] .
\end{aligned}
$$

Then $\Psi_{n}$ and $\Psi_{\circ, n}$ are nonsingular for all $n$ sufficiently large and $\lim _{n \rightarrow \infty} \Psi_{n}=$ $\Psi$ and $\lim _{n \rightarrow \infty} \Psi_{\circ, n}=\Psi_{\circ}$ are finite and nonsingular.

\section{B Appendix: Estimators for $\Psi$ and $\Omega$}

For simplicity of notation we drop subscript $n$ in the following.

\section{B.1 Definition of $G$ and $g$}

Let $\widetilde{\delta}$ be some estimator for $\delta$, let $\widetilde{u}=y-Z \widetilde{\delta}$ be the corresponding estimated residuals, and let $\widetilde{\bar{u}}_{n}=M_{n} \widetilde{u}_{n}, \widetilde{\bar{u}}_{n}=M_{n}^{2} \widetilde{u}_{n}$. Then, $G(\widetilde{\delta})=\left[g_{r s}(\widetilde{\delta})\right]_{r, s=1,2}$ and $g(\widetilde{\delta})=\left[g_{1}(\widetilde{\delta}), g_{2}(\widetilde{\delta})\right]^{\prime}$ are obtained from the expressions for the elements of $\Gamma=\left[\gamma_{r s}\right]_{r, s=1,2}$ and $\gamma=\left[\gamma_{1}, \gamma_{2}\right]^{\prime}$ in (A.1) by suppressing the expectations operator, and replacing the disturbance vectors $u, \bar{u}$, and $\overline{\bar{u}}$ by their predictors $\widetilde{u}, \widetilde{\bar{u}}$, and $\widetilde{\bar{u}}$.

\section{B.2 Definition of $\widetilde{\Psi}$ and $\widetilde{\Omega}$}

Let $\widetilde{u}=y-Z \widetilde{\delta}$ denote the 2SLS residuals, and let $\bar{\rho}$ be some estimator for $\rho$. Then $\widetilde{\Psi}=\left[\widetilde{\psi}_{r s}\right]_{r, s=1,2}$ with

$$
\widetilde{\psi}_{r s}=(2 n)^{-1} \operatorname{tr}\left[\left(A_{r}+A_{r}^{\prime}\right) \widetilde{\Sigma}\left(A_{s}+A_{s}^{\prime}\right) \widetilde{\Sigma}\right]+n^{-1} \widetilde{a}_{r}^{\prime} \widetilde{\Sigma} \widetilde{a}_{s},
$$

where

$$
\begin{aligned}
& \widetilde{\Sigma}=\operatorname{diag}_{i=1, \ldots, n}\left(\widetilde{\varepsilon}_{i}^{2}\right) \quad \widetilde{\varepsilon}=(I-\bar{\rho} M) \widetilde{u} \\
& \widetilde{a}_{r}=\left(I-\bar{\rho} M^{\prime}\right)^{-1} H \widetilde{P} \widetilde{\alpha}_{r} \quad \widetilde{\alpha}_{r}=-n^{-1}\left[Z^{\prime}\left(I-\bar{\rho} M^{\prime}\right)\left(A_{r}+A_{r}^{\prime}\right)(I-\bar{\rho} M) \widetilde{u}\right]
\end{aligned}
$$

and

$$
\widetilde{P}=\left(n^{-1} H^{\prime} H\right)^{-1}\left(n^{-1} H^{\prime} Z\right)\left[\left(n^{-1} Z^{\prime} H\right)\left(n^{-1} H^{\prime} H\right)^{-1}\left(n^{-1} H^{\prime} Z\right)\right]^{-1} .
$$

Let $\widetilde{\Gamma}=G(\widetilde{\delta})$, where $G($.$) is defined in Appendix B.1, and let \widetilde{J}=\widetilde{\Gamma}[1,2 \bar{\rho}]^{\prime}$, then the estimator $\widetilde{\Omega}$ is given by

$$
\begin{aligned}
\widetilde{\Omega} & =\left[\begin{array}{cc}
\widetilde{P}^{\prime} & 0 \\
0 & \left(\widetilde{J}^{\prime} \widetilde{\Psi}^{-1} \widetilde{J}\right)^{-1} \widetilde{J}^{\prime} \widetilde{\Psi}^{-1}
\end{array}\right] \widetilde{\Psi}_{\circ}\left[\begin{array}{cc}
\widetilde{P} & 0 \\
0 & \widetilde{\Psi}^{-1} \widetilde{J}\left(\widetilde{J}^{\prime} \widetilde{\Psi}^{-1} \widetilde{J}\right)^{-1}
\end{array}\right], \\
\widetilde{\Psi}_{\circ} & =\left[\begin{array}{cc}
\widetilde{\Psi}_{\delta \delta} & \widetilde{\Psi}_{\delta \rho} \\
\widetilde{\Psi}_{\delta \rho}^{\prime} & \widetilde{\Psi}
\end{array}\right], \quad \widetilde{\Psi}_{\delta \delta}=n^{-1} H^{\prime}(I-\bar{\rho} M) \widetilde{\Sigma}\left(I-\bar{\rho} M^{\prime}\right) H, \\
\widetilde{\Psi}_{\delta \rho} & =n^{-1} H^{\prime}(I-\bar{\rho} M) \widetilde{\Sigma}\left[\widetilde{a}_{1}, \widetilde{a}_{2}\right] .
\end{aligned}
$$


We will also write $\widetilde{\Psi}(\bar{\rho}), \widetilde{\Omega}(\bar{\rho})$, and $\widetilde{\Psi}_{\circ}(\bar{\rho})$ for $\widetilde{\Psi}, \widetilde{\Omega}$, and $\widetilde{\Psi}_{\circ}$ to explicitly denote the dependence on $\bar{\rho}$.

\section{B.3 Definition of $\widehat{\Psi}$ and $\widehat{\Omega}$}

Let $\widehat{u}=y-Z \widehat{\delta}$ denote the GS2SLS residuals, and let $\bar{\rho}$ be some estimator for $\rho$. Then $\widehat{\Psi}=\left[\widehat{\psi}_{r s}\right]_{r, s=1,2}$ with

$$
\widehat{\psi}_{r s}=(2 n)^{-1} \operatorname{tr}\left[\left(A_{r}+A_{r}^{\prime}\right) \widehat{\Sigma}\left(A_{s}+A_{s}^{\prime}\right) \widehat{\Sigma}\right]+n^{-1} \widehat{a}_{r}^{\prime} \widehat{\mathbf{\Sigma}} \widehat{a}_{s},
$$

where

$$
\begin{array}{ll}
\widehat{\Sigma}=\operatorname{diag}_{i=1, \ldots, n}\left(\widehat{\varepsilon}_{i}^{2}\right) & \widehat{\varepsilon}=(I-\bar{\rho} M) \widehat{u} \\
\widehat{a}_{r}=H \widehat{P} \widehat{\alpha}_{r} & \widehat{\alpha}_{r}=-n^{-1}\left[Z^{\prime}\left(I-\bar{\rho} M^{\prime}\right)\left(A_{r}+A_{r}^{\prime}\right)(I-\bar{\rho} M) \widehat{u}\right]
\end{array}
$$

and

$$
\widehat{P}=\left(n^{-1} H^{\prime} H\right)^{-1}\left(n^{-1} H^{\prime} Z_{*}(\bar{\rho})\right)\left[\left(n^{-1} Z_{*}^{\prime}(\bar{\rho}) H\right)\left(n^{-1} H^{\prime} H\right)^{-1}\left(n^{-1} H^{\prime} Z_{*}(\bar{\rho})\right)\right]^{-1},
$$

and $Z_{*}(\bar{\rho})=Z-\bar{\rho} M Z$. Let $\widehat{\Gamma}=G(\widehat{\delta})$, where $G($.$) is defined in Appendix$ B.1, then the estimator $\widehat{\Omega}$ is given by

$$
\begin{aligned}
& \widehat{\Omega}=\left[\begin{array}{cc}
\widehat{P}^{\prime} & 0 \\
0 & \left(\widehat{J}^{\prime} \widehat{\Psi}^{-1} \widehat{J}\right)^{-1} \widehat{J}^{\prime} \widehat{\Psi}^{-1}
\end{array}\right] \widehat{\Psi}_{\circ}\left[\begin{array}{cc}
\widehat{P} & 0 \\
0 & \widehat{\Psi}^{-1} \widehat{J}\left(\widehat{J}^{\prime} \widehat{\Psi}^{-1} \widehat{J}\right)^{-1}
\end{array}\right], \\
& \widehat{\Psi}_{\circ}=\left[\begin{array}{cc}
\widehat{\Psi}_{\delta \delta} & \widehat{\Psi}_{\delta \rho} \\
\widehat{\Psi}_{\delta \rho}^{\prime} & \widehat{\Psi}
\end{array}\right], \quad \widehat{\Psi}_{\delta \delta}=n^{-1} H^{\prime} \widehat{\Sigma} H, \quad \widehat{\Psi}_{\delta \rho}=n^{-1} H^{\prime} \widehat{\Sigma}\left[\widehat{a}_{1}, \widehat{a}_{2}\right] .
\end{aligned}
$$

We will also write $\widehat{\Psi}(\bar{\rho}), \widehat{\Omega}(\bar{\rho})$, and $\widehat{\Psi}_{\circ}(\bar{\rho})$ for $\widehat{\Psi}, \widehat{\Omega}$, and $\widehat{\Psi}_{\circ}$ to explicitly denote the dependence on $\bar{\rho}$.

\section{Appendix: Proofs}

Proof of Theorem 1: Consider the 2SLS residuals $\widetilde{u}_{n}=y_{n}-Z_{n} \widetilde{\delta}_{n}$. Then clearly $\widetilde{u}_{n}-u_{n}=D_{n} \Delta_{n}$ with $D_{n}=-Z_{n}$ and $\Delta_{n}=\widetilde{\delta}_{n}-\delta$. Next observe that under our Assumptions 1-3 and 4-6, Assumptions 1-3 and 8-10 in Kelejian and Prucha (2007b) clearly hold. Since $\beta$ does not vary with $n$ it now follows directly from Lemma 3 in Kelejian and Prucha (2007) that the fourth moments of the elements of $D_{n}=-Z_{n}$ are uniformly bounded, that Assumption 6 in Kelejian and Prucha (2007b) holds, and:

(a) $n^{1 / 2}\left(\widetilde{\delta}_{n}-\delta\right)=n^{-1 / 2} T_{n}^{\prime} \varepsilon_{n}+o_{p}(1)$ with $T_{n}=F_{n} P$ and where

$$
\begin{aligned}
P & =Q_{H H}^{-1} Q_{H Z}\left[Q_{H Z}^{\prime} Q_{H H}^{-1} Q_{H Z}\right]^{-1}, \\
F_{n} & =\left(I_{n}-\rho M_{n}^{\prime}\right)^{-1} H_{n} .
\end{aligned}
$$


(b) $n^{-1 / 2} T_{n}^{\prime} \varepsilon_{n}=O_{p}(1)$.

(c) $P=O_{p}(1)$ and $\widetilde{P}_{n}-P=o_{p}(1)$ for

$$
\begin{aligned}
& \widetilde{P}_{n}=\left(n^{-1} H_{n}^{\prime} H_{n}\right)^{-1}\left(n^{-1} H_{n}^{\prime} Z_{n}\right) \times \\
& \quad\left[\left(n^{-1} Z_{n}^{\prime} H_{n}\right)\left(n^{-1} H_{n}^{\prime} H_{n}\right)^{-1}\left(n^{-1} H_{n}^{\prime} Z_{n}\right)\right]^{-1} .
\end{aligned}
$$

From this we see that also Assumptions 4 and 7 in Kelejian and Prucha (2007b) are satisfied.

By Assumption A.1 we have $\Gamma_{n}$ is nonsingular for all $n$ sufficiently large and $\lim _{n \rightarrow \infty} \Gamma_{n}=\Gamma$ is finite and nonsingular. Consequently the $\lambda_{\min }\left(\Gamma_{n}^{\prime} \Gamma_{n}\right) \geq$ const $>0$ for $n$ sufficiently large and thus also Assumption 5(a) in Kelejian and Prucha (2007b) holds. Furthermore observe that for $\widetilde{\Upsilon}_{n}=\Upsilon_{n}=I_{2}$ also Assumption 5(b),(c) in Kelejian and Prucha (2007b) are trivially satisfied.

By Assumption A.2 $\Psi_{n}$ and $\Psi_{\circ, n}$ are nonsingular for all $n$ sufficiently large and $\lim _{n \rightarrow \infty} \Psi_{n}=\Psi$ and $\lim _{n \rightarrow \infty} \Psi_{\circ, n}=\Psi_{\circ}$ are finite and nonsingular, and thus the smallest [largest] eigenvalues of $\Psi_{n}, \Psi_{n}^{-1}, \Psi_{\circ, n}$ and $\Psi_{\circ, n}^{-1}$ are bounded away from zero [bounded from above] for sufficiently large $n$.

It now follows immediately from Theorems 1-3 in Kelejian and Prucha (2007b) that the initial GMM estimator for $\rho, \check{\rho}_{n}$, is $n^{1 / 2}$ - consistent and that $\operatorname{plim}_{n \rightarrow \infty} \widetilde{\Psi}_{n}\left(\check{\rho}_{n}\right)=\Psi$ and $\operatorname{plim}_{n \rightarrow \infty} \widetilde{\Psi}_{n}^{-1}\left(\check{\rho}_{n}\right)=\Psi^{-1}$.

The estimator $\widetilde{\rho}_{n}$ is a special case of the GMM estimators for $\rho$ defined in equation (9) in Kelejian and Prucha (2007b) with $\widetilde{\Upsilon}_{n}=\widetilde{\Psi}_{n}^{-1}\left(\widetilde{\rho}_{n}\right)$ and $\Upsilon_{n}=\Psi_{n}^{-1}$. Recalling that the smallest [largest] eigenvalues of $\Psi_{n}^{-1}$ bounded away from zero [bounded from above] for sufficiently large $n$ we see from Theorem 3 in Kelejian and Prucha (2007b) that also in this case Assumption $5(\mathrm{~b}),(\mathrm{c})$ in that paper are satisfied. All other assumptions maintained by Theorems 1-3 in Kelejian and Prucha (2007b) have already been verified, which establishes $n^{1 / 2}$-consistency of $\widetilde{\rho}_{n}$ and its asymptotic efficiency.

The joint limiting distribution of $n^{1 / 2}\left(\widetilde{\delta}_{n}-\delta\right)$ and $n^{1 / 2}\left(\widetilde{\rho}_{n}-\rho\right)$ given by the theorem now follows immediately from Theorem 4 in Kelejian and Prucha (2007b).

\section{Proof of Theorem 2:}

Consider the GS2SLS residuals $\widehat{u}_{n}=y_{n}-Z_{n} \widehat{\delta}_{n}$. Then clearly $\widehat{u}_{n}-u_{n}=$ $D_{n} \Delta_{n}$ with $D_{n}=-Z_{n}$ and $\Delta_{n}=\widehat{\delta}_{n}-\delta$. As in the proof of Theorem 1 , observe that under our Assumptions 1-3 and 4-6, Assumptions 1-3 and 8-10 in Kelejian and Prucha (2007b) clearly hold. Also recall that in the proof of Theorem 1 we have established that the fourth moments of the elements of $D_{n}=-Z_{n}$ are uniformly bounded, and that Assumption 6 in Kelejian and Prucha (2007b) holds. It now follows from Lemma 4 in Kelejian and Prucha (2007b) that ${ }^{6}$

(a) $n^{1 / 2}\left(\widehat{\delta}_{n}\left(\widetilde{\rho}_{n}\right)-\delta\right)=n^{-1 / 2} T_{n}^{\prime} \varepsilon_{n}+o_{p}(1)$ with $T_{n}=F_{n} P$ and where

$$
\begin{aligned}
P & =Q_{H H}^{-1} Q_{H Z^{*}}(\rho)\left[Q_{H Z^{*}}^{\prime}(\rho) Q_{H H}^{-1} Q_{H Z^{*}}(\rho)\right]^{-1}, \\
F_{n} & =H_{n} .
\end{aligned}
$$

\footnotetext{
${ }^{6}$ The argument, and hence the Theorem, also holds if $\widetilde{\rho}_{n}$ is replaced by any other $n^{1 / 2}$-consistent estimator for $\rho$.
} 
(b) $n^{-1 / 2} T_{n}^{\prime} \varepsilon_{n}=O_{p}(1)$.

(c) $P=O_{p}(1)$ and $\widetilde{P}_{n}-P=o_{p}(1)$ for

$$
\begin{aligned}
& \widetilde{P}_{n}=\left(n^{-1} H_{n}^{\prime} H_{n}\right)^{-1}\left(n^{-1} H_{n}^{\prime} Z_{n}^{*}\left(\widetilde{\rho}_{n}\right)\right) \times \\
& \quad\left[\left(n^{-1} Z_{n}^{*}\left(\widetilde{\rho}_{n}\right)^{\prime} H_{n}\right)\left(n^{-1} H_{n}^{\prime} H_{n}\right)^{-1}\left(n^{-1} H_{n}^{\prime} Z_{n}\left(\widetilde{\rho}_{n}\right)\right)\right]^{-1} .
\end{aligned}
$$

From this we see that Assumptions 4 and 7 in Kelejian and Prucha (2007b) are also satisfied.

By Assumption A.1 we have $\Gamma_{n}$ is nonsingular for all $n$ sufficiently large and $\lim _{n \rightarrow \infty} \Gamma_{n}=\Gamma$ is finite and nonsingular. Consequently the $\lambda_{\min }\left(\Gamma_{n}^{\prime} \Gamma_{n}\right) \geq$ const $>0$ for $n$ sufficiently large and thus Assumption 5(a) in Kelejian and Prucha (2007b) also holds.

By Assumption A.3 $\Psi_{n}$ and $\Psi_{\circ, n}$ are nonsingular for all $n$ sufficiently large and $\lim _{n \rightarrow \infty} \Psi_{n}=\Psi$ and $\lim _{n \rightarrow \infty} \Psi_{\circ, n}=\Psi_{\circ}$ are finite and nonsingular, and thus the smallest [largest] eigenvalues of $\Psi_{n}, \Psi_{n}^{-1}, \Psi_{\circ, n}$ and $\Psi_{\circ, n}^{-1}$ are bounded away from zero [bounded from above] for sufficiently large $n$.

The estimator $\widehat{\rho}_{n}$ is a special case of the GMM estimators for $\rho$ defined in equation (9) in Kelejian and Prucha (2007b) with $\widetilde{\Upsilon}_{n}=\widehat{\Psi}_{n}^{-1}\left(\widetilde{\rho}_{n}\right)$ and $\Upsilon_{n}=\Psi_{n}^{-1}$. As remarked above, the smallest [largest] eigenvalues of $\Psi_{n}^{-1}$ bounded away from zero [bounded from above] for sufficiently large $n$, and thus we see from Theorem 3 in Kelejian and Prucha (2007b) that in this case Assumption 5(b),(c) in that paper are also satisfied. All other assumptions maintained by Theorems 1-3 in Kelejian and Prucha (2007b) have already been verified, which establishes $n^{1 / 2}$-consistency of $\widehat{\rho}_{n}$ and its asymptotic efficiency.

The joint limiting distribution of $n^{1 / 2}\left(\widehat{\delta}_{n}-\delta\right)$ and $n^{1 / 2}\left(\widehat{\rho}_{n}-\rho\right)$ given by the theorem now follows immediately from Theorem 4 in Kelejian and Prucha (2007b). 


\section{References}

[1] Anselin, L., Spatial Econometrics: Methods and Models. Boston: Kluwer Academic Publishers, 1988.

[2] Anselin, L. and Florax, R., New Directions in Spatial Econometrics. London: Springer, 1995.

[3] Anselin, L. and Le Gallo, J., Interpolation of Air Quality Measures in Hedonic House Price Models: Spatial Aspects. Spatial Economic Analysis, Taylor and Francis Journals, 1(1) (2006), 31-52.

[4] Audretsch, D.B. and Feldmann, M.P., R\&D Spillovers and the Geography of Innovation and Production. American Economic Review 86 (1996), 630-640.

[5] Baltagi, B.H., Egger, P. and Pfaffermayr, M. Estimating Models of Complex FDI: Are There Third-Country Effects? Journal of Econometrics 140 (2007), 260-281.

[6] Bell, K.P. and Bockstael, N.E., Applying the Generalized-Moments Estimation Approach to Spatial Problems Involving Microlevel Data. Review of Economics and Statistics 82 (2000), 72-82.

[7] Betrand, M., Luttmer, E.F.P., and Mullainathan, S., Net-work Effects and Welfare Cultures. Quarterly Journal of Economics 115 (2000), 10191055 .

[8] Besley, T. and Case, A., Incumbent Behavior: Vote-Seeking, TaxSetting, and Yardstick Competition. American Economic Review 85 (1995), 25-45.

[9] Case, A., Hines Jr., J. and Rosen, H., Budget Spillovers and Fiscal Policy Independence: Evidence from the States. Journal of Public Economics 52 (1993), 285-307.

[10] Cliff, A. and Ord, J., Spatial Autocorrelation, London: Pion, 1973.

[11] Cliff, A. and Ord, J., Spatial Processes, Models and Applications. London: Pion, 1981.

[12] Cohen, J.P, and Morrison Paul, C.J., Airport Infrastructure Spillovers in a Network System. Journal of Urban Economics, 54 (2003) 459-473.

[13] Cohen, J.P, and Morrison Paul, C.J., Public Infrastructure Investment, Interstate Spatial Spillovers, and Manufacturing Cost, Review of Economic and Statistics 86 (2004), 551-560. 
[14] Cohen, J. and Morrison Paul, C.J, The Impacts of Transportation Infrastructure on Property Values: A Higher-Order Spatial Econometric Approach. Journal of Regional Science 47 (2007), 457-478.

[15] Cressie, N.A.C., Statistics of Spatial Data. New York: Wiley, 1993.

[16] Holtz-Eakin, D., Public Sector Capital and the Productivity Puzzle. Review of Economics and Statistics 76 (1994), 12-21.

[17] Kelejian, H.H. and Prucha, I.R., A Generalized Spatial Two-Stage Least Squares Procedure for Estimating a Spatial Autoregressive Model with Autoregressive Disturbances. Journal of Real Estate Finance and Economics 17 (1998), 99-121.

[18] Kelejian, H.H. and Prucha, I.R., A Generalized Moments Estimator for the Autoregressive Parameter in a Spatial Model. International Economic Review 40 (1999), 509-533.

[19] Kelejian, H.H. and Prucha, I.R., Estimation of Simultaneous Systems of Spatially Interrelated Cross Sectional Equations. Journal of Econometrics, 118 (2004), 27-50.

[20] Kelejian, H.H. and Prucha, I.R., HAC Estimation in a Spatial Framework. Journal of Econometrics 140 (2007a), 131-154.

[21] Kelejian, H.H. and Prucha, I.R., Specification and Estimation of Spatial Autoregressive Models with Autoregressive and Heteroskedastic Disturbances. Department of Economics, University of Maryland, 2007b, forthcoming in Journal of Econometrics.

[22] Kelejian, H.H. and D. Robinson, 1995, Spatial correlation: A suggested alternative to the autoregressive model. In L. Anselin and R. Florax, eds., New Directions in Spatial Econometrics (Springer, New York), 7595 .

[23] Keller, W. and Shiue, C. The Origin of Spatial Interaction. Journal of Econometrics 140 (2007), 304-332.

[24] Le Gallo, J. and Dall'erba, S., Evaluating the Temporal and Spatial Heterogeneity of the European Convergence Process, 1980-1999. Journal of Regional Science, 46 (2006) 269-288.

[25] Lee, L.-F., Asymptotic Distributions of Maximum Likelihood Estimators for Spatial Autoregressive Models. Econometrica 72 (2004), 18991925.

[26] Sacredote, B., Peer Effects with random Assignment: Results of Dartmouth Roommates. Quarterly Journal of Economics 116 (2001), 681704 .

[27] Shroder, M., Games the States Don't Play: Welfare Benefits and the Theory of Fiscal Federalism. Review of Economics and Statistics 77 (1995), 183-191. 
[28] Topa, G., Social Interactions, Local Spillovers and Unemployment. Review of Economic Studies 68 (2001), 261-295. 


\section{CESifo Working Paper Series}

for full list see www.cesifo-group.org/wp

(address: Poschingerstr. 5, 81679 Munich, Germany, office@cesifo.de)

2421 Kamila Fialová and Ondřej Schneider, Labour Market Institutions and their Effect on Labour Market Performance in the New EU Member Countries, October 2008

2422 Alexander Ludwig and Michael Reiter, Sharing Demographic Risk - Who is Afraid of the Baby Bust?, October 2008

2423 Doina Maria Radulescu and Michael Stimmelmayr, The Welfare Loss from Differential Taxation of Sectors in Germany, October 2008

2424 Nikolaus Wolf, Was Germany ever United? Evidence from Intra- and International Trade 1885 - 1933, October 2008

2425 Bruno S. Frey, David A. Savage and Benno Torgler, Noblesse Oblige? Determinants of Survival in a Life and Death Situation, October 2008

2426 Giovanni Facchini, Peri Silva and Gerald Willmann, The Customs Union Issue: Why do we Observe so few of them?, October 2008

2427 Wido Geis, Silke Uebelmesser and Martin Werding, Why go to France or Germany, if you could as well go to the UK or the US? Selective Features of Immigration to four major OECD Countries, October 2008

2428 Geeta Kingdon and Francis Teal, Teacher Unions, Teacher Pay and Student Performance in India: A Pupil Fixed Effects Approach, October 2008

2429 Andreas Haufler and Marco Runkel, Firms' Financial Choices and Thin Capitalization Rules under Corporate Tax Competition, October 2008

2430 Matz Dahlberg, Heléne Lundqvist and Eva Mörk, Intergovernmental Grants and Bureaucratic Power, October 2008

2431 Alfons J. Weichenrieder and Tina Klautke, Taxes and the Efficiency Costs of Capital Distortions, October 2008

2432 Andreas Knabe and Ronnie Schöb, Minimum Wage Incidence: The Case for Germany, October 2008

2433 Kurt R. Brekke and Odd Rune Straume, Pharmaceutical Patents: Incentives for R\&D or Marketing?, October 2008

2434 Scott Alan Carson, Geography, Insolation, and Institutional Change in $19^{\text {th }}$ Century African-American and White Stature in Southern States, October 2008

2435 Emilia Del Bono and Daniela Vuri, Job Mobility and the Gender Wage Gap in Italy, October 2008 
2436 Marco Angrisani, Antonio Guarino, Steffen Huck and Nathan Larson, No-Trade in the Laboratory, October 2008

2437 Josse Delfgaauw and Robert Dur, Managerial Talent, Motivation, and Self-Selection into Public Management, October 2008

2438 Christian Bauer and Wolfgang Buchholz, How Changing Prudence and Risk Aversion Affect Optimal Saving, October 2008

2439 Erich Battistin, Clara Graziano and Bruno Parigi, Connections and Performance in Bankers' Turnover: Better Wed over the Mixen than over the Moor, October 2008

2440 Erkki Koskela and Panu Poutvaara, Flexible Outsourcing and the Impacts of Labour Taxation in European Welfare States, October 2008

2441 Marcelo Resende, Concentration and Market Size: Lower Bound Estimates for the Brazilian Industry, October 2008

2442 Giandomenico Piluso and Roberto Ricciuti, Fiscal Policy and the Banking System in Italy. Have Taxes, Public Spending and Banks been Procyclical in the Long-Run? October 2008

2443 Bruno S. Frey and Katja Rost, Do Rankings Reflect Research Quality?, October 2008

2444 Guglielmo Maria Caporale, Antoaneta Serguieva and Hao Wu, Financial Contagion: Evolutionary Optimisation of a Multinational Agent-Based Model, October 2008

2445 Valentina Bosetti, Carlo Carraro and Massimo Tavoni, Delayed Participation of Developing Countries to Climate Agreements: Should Action in the EU and US be Postponed?, October 2008

2446 Alexander Kovalenkov and Xavier Vives, Competitive Rational Expectations Equilibria without Apology, November 2008

2447 Thiess Buettner and Fédéric Holm-Hadulla, Cities in Fiscal Equalization, November 2008

2448 Harry H. Kelejian and Ingmar R. Prucha, Specification and Estimation of Spatial Autoregressive Models with Autoregressive and Heteroskedastic Disturbances, November 2008

2449 Jan Bouckaert, Hans Degryse and Thomas Provoost, Enhancing Market Power by Reducing Switching Costs, November 2008

2450 Frank Heinemann, Escaping from a Combination of Liquidity Trap and Credit Crunch, November 2008

2451 Dan Anderberg, Optimal Policy and the Risk Properties of Human Capital Reconsidered, November 2008 
2452 Christian Keuschnigg and Evelyn Ribi, Outsourcing, Unemployment and Welfare Policy, November 2008

2453 Bernd Theilen, Market Competition and Lower Tier Incentives, November 2008

2454 Ondřej Schneider, Voting in the European Union - Central Europe's Lost Voice, November 2008

2455 Oliver Lorz and Gerald Willmann, Enlargement versus Deepening: The Trade-off Facing Economic Unions, November 2008

2456 Alfons J. Weichenrieder and Helen Windischbauer, Thin-Capitalization Rules and Company Responses, Experience from German Legislation, November 2008

2457 Andreas Knabe and Steffen Rätzel, Scarring or Scaring? The Psychological Impact of Past Unemployment and Future Unemployment Risk, November 2008

2458 John Whalley and Sean Walsh, Bringing the Copenhagen Global Climate Change Negotiations to Conclusion, November 2008

2459 Daniel Mejía, The War on Illegal Drugs in Producer and Consumer Countries: A Simple Analytical Framework, November 2008

2460 Carola Frydman, Learning from the Past: Trends in Executive Compensation over the Twentieth Century, November 2008

2461 Wolfgang Ochel, The Political Economy of Two-tier Reforms of Employment Protection in Europe, November 2008

2462 Peter Egger and Doina Maria Radulescu, The Influence of Labor Taxes on the Migration of Skilled Workers, November 2008

2463 Oliver Falck, Stephan Heblich and Stefan Kipar, The Extension of Clusters: Differencein-Differences Evidence from the Bavarian State-Wide Cluster Policy, November 2008

2464 Lei Yang and Keith E. Maskus, Intellectual Property Rights, Technology Transfer and Exports in Developing Countries, November 2008

2465 Claudia M. Buch, The Great Risk Shift? Income Volatility in an International Perspective, November 2008

2466 Walter H. Fisher and Ben J. Heijdra, Growth and the Ageing Joneses, November 2008

2467 Louis Eeckhoudt, Harris Schlesinger and Ilia Tsetlin, Apportioning of Risks via Stochastic Dominance, November 2008

2468 Elin Halvorsen and Thor O. Thoresen, Parents' Desire to Make Equal Inter Vivos Transfers, November 2008 
2469 Anna Montén and Marcel Thum, Ageing Municipalities, Gerontocracy and Fiscal Competition, November 2008

2470 Volker Meier and Matthias Wrede, Reducing the Excess Burden of Subsidizing the Stork: Joint Taxation, Individual Taxation, and Family Splitting, November 2008

2471 Gunther Schnabl and Christina Ziegler, Exchange Rate Regime and Wage Determination in Central and Eastern Europe, November 2008

2472 Kjell Erik Lommerud and Odd Rune Straume, Employment Protection versus Flexicurity: On Technology Adoption in Unionised Firms, November 2008

2473 Lukas Menkhoff, High-Frequency Analysis of Foreign Exchange Interventions: What do we learn?, November 2008

2474 Steven Poelhekke and Frederick van der Ploeg, Growth, Foreign Direct Investment and Urban Concentrations: Unbundling Spatial Lags, November 2008

2475 Helge Berger and Volker Nitsch, Gotcha! A Profile of Smuggling in International Trade, November 2008

2476 Robert Dur and Joeri Sol, Social Interaction, Co-Worker Altruism, and Incentives, November 2008

2477 Gaëtan Nicodème, Corporate Income Tax and Economic Distortions, November 2008

2478 Martin Jacob, Rainer Niemann and Martin Weiss, The Rich Demystified - A Reply to Bach, Corneo, and Steiner (2008), November 2008

2479 Scott Alan Carson, Demographic, Residential, and Socioeconomic Effects on the Distribution of $19^{\text {th }}$ Century African-American Stature, November 2008

2480 Burkhard Heer and Andreas Irmen, Population, Pensions, and Endogenous Economic Growth, November 2008

2481 Thomas Aronsson and Erkki Koskela, Optimal Redistributive Taxation and Provision of Public Input Goods in an Economy with Outsourcing and Unemployment, December 2008

2482 Stanley L. Winer, George Tridimas and Walter Hettich, Social Welfare and Coercion in Public Finance, December 2008

2483 Bruno S. Frey and Benno Torgler, Politicians: Be Killed or Survive, December 2008

2484 Thiess Buettner, Nadine Riedel and Marco Runkel, Strategic Consolidation under Formula Apportionment, December 2008

2485 Irani Arraiz, David M. Drukker, Harry H. Kelejian and Ingmar R. Prucha, A Spatial Cliff-Ord-type Model with Heteroskedastic Innovations: Small and Large Sample Results, December 2008 